\title{
$L_{\infty \lambda}$-EQUTVALENCE, ISOMORPHISM AND POTENTIAL ISOMORPHISM(') \\ BY
}

MARK NADEL AND JONATHAN STAVI

\begin{abstract}
ABSTRACr. It is well known that two structures are $L_{\infty}$ wequivalent iff they are potentially isomorphic [that is, isomorphic in some (Cohen) extension of the universe]. We prove that no characterization of $L_{\infty} \lambda_{\text {-equivalence along }}$ these lines is possible (at least for successor cardinals $\lambda$ ) and the potentialisomorphism relation that naturally comes to mind in connection with $L_{\infty} \lambda$ is often not even transitive and never characterizes $\equiv_{\infty \lambda}$ for $\lambda>\omega$. A major part of the work is the construction of $k^{+}$-like linear orderings (also Boolean algebras) A, B such that $N_{k+}(\mathrm{A}, \mathrm{B})$, where $N_{\lambda}(\mathrm{A}, B)$ means: $\mathrm{A}$ and $\mathrm{B}$ are nonisomorphic $L_{\infty} \lambda$-equivalent structures of cardinality $\lambda$.
\end{abstract}

Introduction. Let $\mathbf{M}=\langle M, \varepsilon\rangle$ be a countable transitive $\varepsilon$-model of ZFC. When referring to extensions $\mathbf{N}=\langle N, \varepsilon\rangle$ of $\mathbf{M}$ we always mean extensions that are also transitive $\varepsilon$-models. It is a simple consequence of Karp's back-and-forth characterization of $L_{\infty}$-equivalence that if $\mathbf{N} \supseteq \mathbf{M}$ and $\mathbf{A}, \mathbf{B}$ $\in M$ are structures of the same similarity type then

$$
\mathbf{A} \equiv_{\infty \omega} \mathbf{B} \text { in } \mathbf{M} \text { iif } \mathbf{A} \equiv_{\infty \omega \omega} \mathbf{B} \text { in } \mathbf{N} \text {. }
$$

This is shown, for example in the expository article [Ba2] with which we assume familiarity.

From the absoluteness result (*) and the fact that any two countable $L_{\infty}$-equivalent structures are isomorphic the following theorem follows easily. lent:

0.1. ThEOREM. For structures $\mathrm{A}, \mathrm{B} \in M$ the following conditions are equiva-

(1) $\mathbf{A}$ and $\mathbf{B}$ are isomorphic in some extension of $\mathbf{M}$;

(2) $\mathbf{A} \equiv_{\infty \omega \omega} \mathbf{B}$ in $\mathbf{M}$;

(3) $\mathbf{A}$ and $\mathbf{B}$ are isomorphic in every extension $\mathbf{N}$ of $\mathbf{M}$ such that $\mathbf{N}=|A|<$ $\kappa_{0} \&|B|<\aleph_{0}$.

(4) $\mathbf{A}$ and $\mathbf{B}$ are isomorphic in some Cohen extension of $\mathbf{M}$.

[Show that $(1) \Rightarrow(2) \Rightarrow(3) \Rightarrow(4) \Rightarrow(1)$.]

A suggestive way of expressing (1) is to say that A and B are potentially isomorphic over $\mathbf{M}$. Note that we could add the equivalent conditions:

$\left(1^{\prime}\right) A \cong B$ (in the "real universe");

Received by the editors October 15, 1975.

AMS (MOS) subject classifications (1970). Primary 02H10; Secondary 02K05, $02 \mathrm{H} 13$.

(') The main results of this paper were announced in [NS]. 
(2') $A \equiv_{\infty \omega \omega} B$ (in the "real universe").

However, for the generalization we propose to study, no analogues of $\left(1^{\prime}\right),\left(2^{\prime}\right)$ will play any role.

Now let $\lambda$ be some infinite cardinal of $M$. Is it possible to characterize $L_{\infty \lambda}$-equivalence in $M$ in an analogous way, as isomorphism in some or all extensions of $\mathbf{M}$ satisfying some conditions, at least for a large natural class of models?

The most natural constraint to put on $\mathbf{N}$ is that $N$ contains no new sequences of elements of $M$ of length $\left\langle\lambda\right.$. In symbols, $N \cap^{\alpha} M \subseteq M$ for each $\alpha<\lambda$, or more briefly: $N \cap^{<\lambda} M \subsetneq M$. This implies that $\lambda$ remains a cardinal in $\mathbf{N}$ and that the notions of being an $L_{\infty \lambda}$-formula and of such a formula being satisfied in a structure are absolute between $\mathbf{M}$ and $\mathbf{N}$. Using the natural generalization of the back-and-forth characterization from $L_{\infty}$ to $L_{\infty \lambda}$ one sees that even the notion $\equiv_{\infty \lambda}$ is absolute between $\mathbf{M}$ and $\mathbf{N}$, that is, we have the analogue of (*).

Let us call two structures $\mathbf{A}, \mathbf{B} \in M \lambda$-potentially isomorphic over $\mathbf{M}$ (written: $\mathbf{A}(\lambda-\mathrm{PI}) \mathbf{B}$ over $\mathbf{M}$ ) when $\mathbf{A}$ and $\mathbf{B}$ are isomorphic in some extension $\mathrm{N}$ of $\mathrm{M}$ satisfying $N \cap^{<\lambda} M \subseteq M$. In view of Theorem 0.1 it would be nice to have the equivalence of:

(1 $1_{\lambda}$ A $(\lambda$-PI)B over $\mathbf{M}$;

$\left(2_{\lambda}\right) A \equiv \equiv_{\infty \lambda} B$ in $\mathbf{M}$;

(3) $A \cong B$ in every extension $N$ of $M$ such that

$$
N \cap^{<\lambda} M \subseteq M, \quad \mathrm{~N} \vDash "|A|<\lambda \&|B|<\lambda " .
$$

$\left(4_{\lambda}\right) A \simeq B$ in some Cohen extension $N$ of $M$ such that

$$
N \cap<\lambda M \subseteq M \text {. }
$$

The proof of Theorem 0.1 breaks down here in the step $\left(2_{\lambda}\right) \Rightarrow\left(3_{\lambda}\right)$. The reason is that the statement "any two $L_{\infty \lambda}$-equivalent structures of cardinality $<\lambda$ are isomorphic" is false except when $\lambda$ has cofinality $\omega$ (more on this in $\S 1)$. Also the step $\left(3_{\lambda}\right) \Rightarrow\left(4_{\lambda}\right)$ can be done (by a standard forcing construction) only for regular $\lambda$, so that there is no value of $\lambda$ other than $\omega$ for which the whole proof goes through.

The aim of this paper is to study the relation " $\lambda$-PI" and related notions of potential isomorphism; more generally we shall inquire whether any notion of this type can characterize $\equiv_{\infty \lambda}$. As is clear from the last paragraph these questions are related to the existence of structures $\mathbf{A}, \mathbf{B}$ such that $N_{\lambda}(\mathbf{A}, \mathbf{B})$ (see Abstract above). Thus we are led to construct some new examples of such structures, especially linear orderings. Our main results concerning potential isomorphisms are negative.

Here is a summary of the contents and main results of the work: In $\$ 1$ we review some known (but not commonly well-known) background results, including an old unpublished example of trees $\mathrm{A}, \mathrm{B}$ such that $N_{\lambda}(\mathrm{A}, \mathrm{B})$ due to M. Morley. \$2 contains two main negative results, both based partly on an analysis of the Morley example. Theorem 2.3 states that the equivalence 
$(1)_{\lambda} \Leftrightarrow\left(2_{\lambda}\right)$ considered above is in fact false for all $\lambda>\omega$ in $\mathbf{M}$ (obviously $\left[\left(1_{\lambda}\right) \Rightarrow\left(2_{\lambda}\right)\right]$ is true). Theorem 2.5 says that no notion of potential isomorphism over $\mathbf{M}$ (in a precisely defined sense) can characterize $L_{\infty} \lambda^{-}$ equivalence in $\mathbf{M}$, at least for successor cardinals. In $\$ 3$ we present and explain an example of $\lambda$-like orderings A, B such that $N_{\omega_{1}}(\mathrm{~A}, \mathrm{~B})$ suggested by $J$. Hutchinson [Hu] to prove that the relation " $\omega_{1}-P I$ over $M$ " is not transitive. In $\$ 4$ we generalize this construction to $\kappa^{+}$-like orderings for regular $\kappa$. (A $\lambda$-like ordering is a linear ordering of cardinality $\lambda$ in which every element has $<\lambda$ predecessors.) The main result (depending partly on some unpublished set-theoretic work of Gregory-Harrington [GH] and [St1]) is that the relation " $\lambda$-PI over $M$ " is not transitive if $\lambda$ has (in M) the form $\lambda=\kappa^{+}$ with $\kappa=\kappa^{k}$. In $\$ 5$ we construct $\kappa^{+}$-like orderings A, B such that $N_{\kappa^{+}}(\mathrm{A}, \mathrm{B})$ also for singular $\kappa$, assuming that $V=L$ (and using Jensen's [Jn] principle $\square_{K}$ ). In $\$ 6$ we show that all previous negative results remain true when structures are restricted to be Boolean algebras. In $\$ 7$ we study a stronger relation of potential isomorphism $\left(\lambda-\mathrm{PI}^{*}\right.$ over $\left.\mathbf{M}\right)$ which is transitive and for which an analogue of the equivalence (1) $\Leftrightarrow(3) \Leftrightarrow(4)$ of Theorem 0.1 is valid. Since this relation is transitive it might characterize $\equiv_{\mathfrak{C}}$ for some interesting abstract logic $\mathcal{L}$ (stronger than $L_{\infty \lambda}$ ). The question whether this is the case and related questions are suggested in the concluding section $\$ 8$.

Acknowledgement. The authors would like to thank Professors C. C. Chang, D. Kueker and J. B. Paris for help in collecting the information given in $\$ 1$, and M. Magidor who contributed the proof of Lemma 5.4.

1. Review of known results. We assume acquaintance with the expository paper [Ba2] or equivalent material. It will also help to be familiar with $\$ 1$ of [Ch].

The back-and-forth characterization of $\equiv_{\infty \omega}$ (due to Karp) can be extended in a straightforward way to $L_{\infty \lambda}$ as follows (this has been observed independently by many people; it appears already in [Be]). Let $I: A \approx_{\lambda} B$ mean that $I$ is a nonempty set of partial isomorphisms from $\mathbf{A}$ to $\mathbf{B}$ enjoying the following $<\lambda$-back-and-forth-property: For each $f \in I, X \in P_{<\lambda}(A)$ (i.e. $X \subseteq A,|X|<\lambda)$ there exists $g \in I$ such that $g \supseteq f$, $\operatorname{dom}(g) \supseteq X$; similarly $(\forall f \in I)\left(\forall Y \in P_{<\lambda}(B)\right)(\exists g \in I)[g \supseteq f \&$ range $(g) \supseteq Y]$. Call $\mathbf{A}$ and B $\lambda$-partially isomorphic $\left(A \approx_{\lambda} \mathbf{B}\right)$ when $\exists I\left(I: A \approx_{\lambda} \mathbf{B}\right)$.

\subsection{THEOREM. $\mathbf{A} \equiv_{\infty \lambda} \mathbf{B}$ iff $\mathbf{A} \cong_{\lambda} \mathbf{B}$.}

The proof (given, e.g., in [Ta]) is a straightforward extension of the proof of Karp's theorem (which is the case $\lambda=\omega$ ) given in [Ba2]. [The theorem implies that $L_{\infty \lambda}$-equivalence is absolute between standard models $\mathbf{M} \subseteq \mathbf{N}$ of set theory such that $\mathbf{N} \cap^{<\lambda} M \subseteq M$, where $\lambda$ is a cardinal of $\mathbf{M}$, because satisfaction of $L_{\infty \lambda}$ sentences and the statement " $I: A \simeq_{\lambda} \mathrm{B}$ " are absolute between $\mathbf{M}$ and $\mathbf{N}$.]

The other basic result on $L_{\infty}$ used in the Introduction is that if $|A| \leqslant \aleph_{0}$, 
$|B|<\kappa_{0}$ then $\mathbf{A} \equiv_{\infty \omega} \mathbf{B} \Rightarrow \mathbf{A} \cong \mathbf{B}$. Now if $\mathbf{A} \equiv_{\infty \lambda} \mathbf{B}$ and $|A|<\lambda$ or $|B|<\lambda$ then $A \simeq B$ (trivial). Chang [Ch, \&1] shows that if $\operatorname{cf}(\lambda)=\omega$ and $|A|<\lambda$, $|B|<\lambda$ then $A \equiv_{\infty \lambda} B \Rightarrow A \approx B$ (the proof is a simple application of the back-and-forth method). The main result of [Ta] is that if $\operatorname{cf}(\lambda)>\omega$ then there exist structures $\mathrm{A}, \mathrm{B}$ (with one binary relation) such that $N_{\lambda}(\mathrm{A}, \mathrm{B})$. Recall $N_{\lambda}(\mathrm{A}, \mathrm{B})$ is short for: $|A|=|B|=\lambda, \mathrm{A} \equiv_{\infty \lambda} \mathrm{B}$ and $\mathrm{A} \cong \mathrm{B}$. While Tait's example is very complicated, simpler examples of $A, B$ such that $N_{\lambda}(\mathrm{A}, \mathrm{B})$ for various regular $\lambda>\omega$ had been given before. In fact [GS] implicitly contains an example of $\lambda$-trees A, B such that $N_{\lambda}(\mathrm{A}, \mathrm{B})$, provided that $\lambda$ is of the form $\kappa^{+}$where $\kappa=\kappa^{\Sigma}$. Another example of Morley for $\lambda=\omega_{1}$, which generalized easily to arbitrary. regular $\lambda>\omega$, gives trees $\mathbf{A}, \mathbf{B}$ such that $N_{\lambda}(\mathrm{A}, \mathrm{B})$. This example is mentioned (but not described) by Chang [Ch, §1]. We briefly describe it below.

Paris [Pa] gave examples of linear orderings A, B such that $N_{\omega_{1}}(\mathbf{A}, \mathbf{B})$. A simpler example, which gives $\omega_{1}$-like dense linear orderings is noted by $\mathrm{J}$. Hutchinson [Hu, \$3] $\left.{ }^{2}\right)$; the orderings involved had been studied by $\mathrm{J} . \mathrm{H}$. Conway [Co]. We shall deal with the Conway-Hutchinson examples (which are the simplest known ones) in detail later and generalize them. Several people have studied Abelian groups related by $N_{\lambda}$ to free Abelian groups-cf., e.g., [Ek] and references there; more on groups in [Mk] and [St2].

Here is a brief description of the (generalized) Morley example as simplified by Makkai: Let $\lambda>\omega$ be regular. We define by induction on $n$ trees $T_{n} . T_{1}$ is obtained by associating with each limit ordinal $\alpha<\lambda$ a branch of length $\alpha$, all branches splitting from the same root. Assume $T_{n}$ has been defined. Let

$$
P_{n}=\left\{p \in T_{n} \mid\left\{q \in T_{n} \mid q>p\right\} \text { is linearly ordered by }<T_{n}\right\}
$$

and for each $p \in P_{n}$ let $\Lambda_{n}(p)$ be the order type of $\left\{q \in T_{n} \mid q>p\right\}$ (which is necessarily well ordered). Each $\Lambda_{n}(p)$ will be a limit ordinal $<\lambda$. Now let $T_{n+1}$ be obtained from $T_{n}$ by adjoining to each $p \in P_{n}$ a new branch of length $\alpha$, starting from $p$ for each limit ordinal $\alpha<\lambda, \alpha \neq \Lambda_{n}(p)$. Thus if $p \in P_{n}$ then the successors of $p$ in $T_{n+1}$ form a tree isomorphic to $T_{1}$, with $p$ itself as the root.

Now let $A=\cup_{n} T_{n},<_{A}=\cup_{n}\left\langle T_{n}, \mathbf{A}=\left\langle A,\left\langle_{A}\right\rangle\right.\right.$. Thus $\mathbf{A}$ is a tree with no branch of length $\lambda$ (since $\operatorname{cf}(\lambda)>\omega$ ). To get the other tree B start with a single branch of length $\lambda$ and adjoin to each point $p$ on it a set of new successors which is an isomorphic copy of $\mathbf{A}$ (with the point $p$ itself corresponding to the root). It is clear that $|A|=|B|=\lambda, A \approx B$ and with some work one shows that $\mathrm{A} \simeq_{\lambda} \mathrm{B}$. Thus $N_{\lambda}(\mathrm{A}, \mathrm{B})$ holds. The proof that $\mathrm{A} \simeq_{\lambda} \mathrm{B}$ is where the regularity of $\lambda$ is fully used.

(2) We later learnt that the same example had been observed in 1971 by J. Silver (unpublished). 
2. Remarks on potential isomorphism. At the end of $\S 1$ we have sketched the definition, in ZFC, of two trees $\mathbf{A}_{\lambda}, \mathbf{B}_{\boldsymbol{\lambda}}$ associated with each ordinal $\lambda$ and metioned that

(*)

$$
\text { ZFC ト “ }(\forall \text { regular } \lambda>\omega) N_{\lambda}\left(\mathbf{A}_{\lambda}, \mathbf{B}_{\lambda}\right) \text { ". }
$$

The definition of $A_{\lambda}, B_{\lambda}$ from $\lambda$ is very explicit, hence absolute between standard models of ZFC. Thus if $\mathbf{M}$ is a standard model (= transitive $\varepsilon$-model of $Z F$ C), $\lambda$ a regular uncountable cardinal in $\mathbf{M}$ and $\mathbf{N} \supseteq \mathbf{M}$ then $\mathbf{A}_{\lambda}$, $\mathbf{B}_{\lambda}$ are the same in $\mathbf{M}$ and in $\mathbf{N}$. If $\lambda$ is regular in $\mathbf{N}$ too, it follows from (*) that $\mathbf{A}_{\lambda}, \mathbf{B}_{\lambda}$ are not isomorphic in $\mathbf{N}$. This proves the following (where, throughout this section $\mathbf{M}$ is a fixed standard model of ZFC).

2.1. THEOREM. Let $\lambda$ be a regular uncountable cardinal of $M$. Then there exist trees $\mathbf{A}, \mathbf{B} \in \mathbf{M}$ such that

(i) " $|A|=|B|=\lambda$ and $\mathbf{A} \equiv_{\infty \lambda} \mathbf{B}$ " holds in $\mathbf{M}$;

(ii) $\mathbf{A}, \mathbf{B}$ are not isomorphic in $\mathbf{M}$, nor in any extension $\mathbf{N}$ of $\mathbf{M}$ in which $\lambda$ is regular.

It follows from (ii) that $A, B$ are not $\lambda$-PI over $\mathbf{M}$ in the sense defined in the introduction. Thus for regular $\lambda$ the relation $\lambda$-PI (over $\mathbf{M}$ ) is strictly stronger than $\equiv_{\infty \lambda}$ (in M).

A similar argument using our construction in $\$ 4$ of two $\lambda$-like orderings $\mathbf{A}$, B such that $N_{\lambda}(\mathbf{A}, \mathbf{B})$ for certain $\lambda$ establishes the following:

2.2. Theorem. Let $\kappa$ be a regular cardinal, $\lambda=\kappa^{+}$(in $\left.\mathbf{M}\right)$. There exist $\mathbf{A}$, $\mathbf{B} \in \mathbf{M}$ such that

(i) in $\mathbf{M}, \mathbf{A}$ and $\mathbf{B}$ are $\lambda$-like dense linear orderings and $\mathbf{A} \equiv_{\infty \lambda} \mathbf{B}$;

(ii) $\mathbf{A}$ and $\mathbf{B}$ are not isomorphic in $\mathbf{M}$, nor in any extension $\mathbf{N}$ of $\mathbf{M}$ in which $\kappa$ is regular and $\lambda=\kappa^{+}$(hence $\mathbf{A}$ and $\mathbf{B}$ are not $\lambda$-PI over $\mathbf{M}$ ).

Again, all one has to notice for the proof are certain absoluteness features of the constructions in $\S 4$.

2.3. Theorem. Let $\lambda$ be a cardinal of $\mathbf{M}, \lambda>\omega$. Then there exist $\mathbf{A}, \mathbf{B} \in M$ such that in $\mathbf{M} \mathbf{A} \equiv_{\infty \lambda} \mathbf{B}$ yet $\mathbf{A}$ and $\mathbf{B}$ are not $\lambda$-PI over $\mathbf{M}$.

Proof. For regular $\lambda$ this follows from Theorem 2.1. If $\lambda$ is singular in $\mathbf{M}$ note that if $\mathbf{N}$ is any extension of $\mathbf{M}$ such that $N \cap^{<\lambda} M \subseteq M$ then $N \cap \cap^{<\lambda^{+}} M \subseteq M$ (that is, if there are no new sequences of length $<\lambda$ then there are no new sequences of length $\lambda$, hence not of any length $<\lambda^{+}$; the proof is left to the reader). Thus if $\lambda$ is singular in $\mathbf{M}$ and $\mathbf{A}(\lambda-\mathrm{PI}) \mathbf{B}$ over $\mathbf{M}$ then $\mathbf{A}\left(\lambda^{+}-\mathrm{PI}\right) \mathbf{B}$ over $\mathbf{M}$ hence $\mathbf{A} \equiv_{\infty \lambda^{+}} \mathbf{B}$ in $\mathbf{M}$. Thus to prove the theorem it suffices to find (in $\mathbf{M}) \mathbf{A}, \mathbf{B}$ such that $\mathbf{A} \equiv_{\infty \lambda} \mathbf{B}$ but $\mathbf{A} \equiv_{\infty \lambda}+\mathbf{B}$. Take $\mathbf{A}=\langle A\rangle$, $\mathbf{B}=\langle B\rangle$ where $|A|=\lambda,|B|=\lambda^{+}$. Let $I=\{f \mid f$ is a 1-1 mapping from a subset of $A$ of cardinality $\left\langle\lambda\right.$ into $B$ \}. It is clear that $I: A \approx_{\lambda}$ B hence

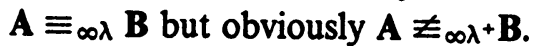

REMARK. In fact an even stronger result holds when $\operatorname{cf}(\lambda)>\omega$. Tait's 
example provides structures $\mathbf{A}$ and $\mathbf{B}$, with $|A|=|B|=\lambda$ in $\mathbf{M}$. This follows since the definition of the structures is sufficiently absolute and since their nonisomorphism depends only on the fact that $\operatorname{cf}(\lambda)>\omega$.

Theorem 2.3 shows that for $\lambda>\omega$ the attempt to characterize $\equiv_{\infty \lambda}$ by means of $\lambda$-PI fails. How about a characterization in terms of some other notion of potential isomorphism? We now show that this, too, is impossible, at least for successor $\lambda$.

2.4. Definition. Let $\mathbf{E}$ be a class of extensions of $\mathbf{M}$ and let $\mathbf{A}, \mathbf{B}$ be structures (of the same similarity type) in $\mathbf{M}$. We say that $\mathbf{A}$ and $\mathbf{B}$ are potentially isomorphic over $M$ relative to $\mathbf{E}\left[\mathbf{A}\left(\mathrm{PI}_{\mathbf{M}}^{\mathrm{E}}\right) \mathbf{B}\right]$ when there exists some $\mathbf{N} \in \mathbf{E}$ such that $\mathbf{A}$ and $\mathbf{B}$ are isomorphic in $\mathbf{N}$.

[For a suitable $\mathbf{E}=\mathbf{E}_{\lambda}, \mathrm{PI}_{\mathbf{M}}^{\mathrm{E}}$ is our old " $\lambda$-PI over $\mathbf{M}$ ".]

2.5. THEOREM. Let $\lambda$ be a successor cardinal of $\mathbf{M}$. There is no class $\mathbf{E}$ of extensions of $\mathbf{M}$ such that for all $\mathbf{A}, \mathbf{B} \in \mathbf{M}: \mathbf{A} \equiv_{\infty \lambda} \mathbf{B}$ in $\mathbf{M}$ iff $\mathbf{A}\left(\mathbf{P I}_{\mathbf{M}}^{\mathbf{E}}\right) \mathbf{B}$.

Proof. Let $\lambda=\kappa^{+}$in $\mathbf{M}$ and let $\mathbf{E}$ be a class of extensions of $\mathbf{M}$. Consider two cases:

(I) In each $\mathbf{N} \in \mathbf{E} \lambda$ is a cardinal. In this case $\lambda$ is a successor cardinal (hence regular) in each $\mathbf{N} \in \mathbf{E}$, since the statement $(\forall \alpha<\lambda)(|\alpha|<|\kappa|)$ holds in every $\mathbf{N} \supseteq \mathbf{M}$ and implies $\lambda<|\kappa|^{+}$. By Theorem 2.1 we have (trees) $A, B \in M$ such that $A \equiv_{\infty \lambda} B$ in $M$ but $A \cong B$ in any $N \in E$ so $A\left(P I_{M}^{E}\right) B$ fails.

(II) There exists $\mathbf{N} \in \mathbf{E}$ in which $\lambda$ is not a cardinal. Let $\mathbf{N}_{0}$ be such an $\mathbf{N}$. $\mathbf{N}_{0}=|\lambda|=|\kappa|$. Take $\mathbf{A}=\langle A\rangle, \mathbf{B}=\langle B\rangle$ where $A, B \in M$ and in $\mathbf{M},|A|=$ $\kappa,|B|=\lambda$. Clearly $A Z_{\infty \lambda} B$ in $M$ but $N_{0}=$ "A $\simeq B$ " hence $A(P I=B$.

We conjecture that Theorem 2.5 holds for every uncountable cardinal $\lambda$ of M.

We now introduce a still more general notion of potential isomorphism.

2.6. Definition. Let $\mathbf{E}$ be a class of extensions of $\mathbf{M}$ and $\mathbf{F}$ a collection of subclasses of $\mathbf{E}$. Let $\mathbf{A}, \mathbf{B}$ be structures in $\mathbf{M}$. We write $\mathbf{A}\left(\mathrm{PI}_{\mathbf{M}}^{\mathbf{E}, \mathbf{F}}\right) \mathbf{B}$ when

$$
(\exists K \in \mathbf{F})(\forall \mathbf{N} \in K) \mathbf{N} \vDash \mathbf{A} \approx \mathbf{B} \text {. }
$$

For example when $\mathbf{E}=\{K \subseteq \mathbf{E} \mid K \neq \varnothing\}$ we get the relation $\mathrm{PI}_{M}^{\mathbf{E}}$ previously defined. Another case of interest is when $\mathbf{F}=\{\mathbf{E}\}$ so that $\mathbf{A}\left(\mathrm{PI}_{\mathbf{M}}^{\mathbf{E}} \mathbf{\mathbf { F }}\right) \mathbf{B}$ means that $\mathbf{A}$ and $\mathbf{B}$ are isomorphic in every member of $\mathbf{E}$. Using the same pairs of models as in the proof of Theorem 2.5 one similarly establishes

2.7. THEOREM. Let $\lambda$ be a successor cardinal of $\mathbf{M}$. There do not exist $\mathbf{E}, \mathbf{F}$ as in 2.6 such that for all $\mathbf{A}, \mathbf{B} \in M$;

$$
\mathbf{A} \equiv \equiv_{\infty \lambda} \mathbf{B} \quad \text { in } \mathbf{M} \text { iff } \mathbf{A}\left(\mathrm{PI}_{\mathbf{M}}^{\mathbf{E}, \mathbf{F}}\right) \mathbf{B} \text {. }
$$

The pair of models used in (II) of the proof of 2.5 may seem less than satisfying. After all, it still might be that $\equiv_{\infty \lambda}$ is equivalent to some $\operatorname{PI}_{\mathbf{M}}^{\mathbf{E}}$ or $\mathrm{PI}_{\mathbf{M}}^{\mathrm{EF}}$ when restricted to some natural large class of structures. With this in 
mind we mention another example for the case $\lambda=2^{\mathrm{N}_{0}}=\omega_{1}$ originally given in [Na1], which is not as easily dismissed. In particular, both $\mathbf{A}$ and $B$ will have cardinality $\lambda$, as well as all their nonempty definable subsets.

The model $\mathbf{A}$ will be the ordering on the reals of $\mathbf{M}$ while $\mathbf{B}$ will be the ordering on the reals without some particular point, say $\mathbf{0}$. Clearly $\mathbf{A}$ and $\mathbf{B}$ are not $\equiv_{\infty \omega_{1}}$ since they can be distinguished by the sentence of $L_{\infty \omega_{1}}$ expressing sequential completeness. On the other hand, if $\mathbf{N}$ is any extension of $\mathbf{M}$ containing a new real, then in $\mathbf{N}, \mathbf{A}$ and $\mathbf{B}$ are isomorphic. To see this, choose in $\mathbf{N}$ a new real $r$ and monotone sequences $\left\langle a_{n}\right\rangle$ and $\left\langle b_{n}\right\rangle$ of rationals strictly increasing to 0 and $r$ respectively. Similarly choose decreasing sequences. $\left\langle c_{n}\right\rangle$ and $\left\langle d_{n}\right\rangle$. Now, piece together an isomorphism from $\mathbf{A}$ to $\mathbf{B}$ by choosing isomorphisms from $\left(-\infty, a_{0}\right]$ to $\left(-\infty, b_{0}\right],\left(a_{0}, a_{1}\right]$ to $\left(b_{0}, b_{1}\right]$, etc. (with intervals denoting sets in $M$ ).

Now, let $\mathbf{A}^{\prime}$ and $\mathbf{B}^{\prime}$ denote the pair of models for $\omega_{1}$ given by the Morley example. As we noted earilier, $A^{\prime}$ and $B^{\prime}$ only become isomorphic in $N$ if $\omega_{1}$ is collapsed. Thus, it is strictly easier to make $\mathbf{A}$ and $\mathbf{B}$ isomorphic than $\mathbf{A}^{\prime}$ and $\mathbf{B}^{\prime}$ even though the latter two are $\equiv_{\infty \omega_{1}}$ while the former are not.

This example has an added feature. Clearly the equivalence or nonequivalence of two structures in $L_{\infty}$ gives some indication of their similarity. On the other hand, so does the relative difficulty of making these structures isomorphic in extensions of $\mathbf{M}$. The proof of Theorem 2.5 shows that the former notion may be more sensitive than the latter. The previous paragraph shows that the two measures of similarity are incompatible.

Returning now to the relation $\lambda$-PI (over M) we may ask whether $|A|=|B|$ $=\lambda \& A(\lambda-\mathrm{PI}) B \Rightarrow \mathrm{A} \cong \mathbf{B}$ (for all $\mathbf{A}, \mathbf{B} \in \mathbf{M}$ ). Also, could the realtion " $\lambda-\mathrm{PI}$

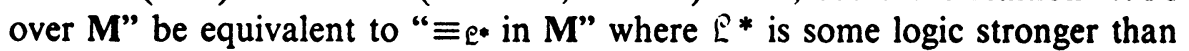
$L_{\infty \lambda}$ ?

We shall give negative answers to both questions at least when $\lambda=\kappa^{+}$, $\kappa=\kappa$, by our generalization of the Hutchinson example. The negative answer to the second question is an immediate consequence of the nontransitivity of the relation $\lambda$-PI, which we shall establish.

In $\$ 7$ we shall study a stronger potential isomorphism relation $\lambda$-PI* for which we have positive results.

3. $\omega_{1}$-like dense linear orderings. It was noted by J. Hutchinson in [Hu] (and earlier by J. Silver) that any two $\omega_{1}$-like dense linear orderings (d.l.o.'s) with a first element are $L_{\infty \omega_{1}}$ equivalent. Indeed, if $D$ and $D^{\prime}$ are such orderings and $I=\left\{f \mid\right.$ there exist initial segments $S$ of $D$ and $S^{\prime}$ of $D^{\prime}$ such that $f$ is an isomorphism of $S$ and $S^{\prime}$, and there is no first element in $D-S$ nor in $\left.D^{\prime}-S^{\prime}\right\}$ then it is easy to see that $I: D \simeq_{\omega_{1}} D^{\prime}$, hence. $D \equiv_{\infty \omega_{1}} D^{\prime}$.

Now let $D_{0}, D_{1}$ be ordered sets of order type $1+\eta \cdot \omega_{1},(1+\eta) \cdot \omega_{1}$ respectively ( $\eta$ is the order type of rational numbers). By the above $D_{0}$ $\equiv_{\infty \omega_{1}} D_{1}$. However in $D_{1}$ there is an increasing continuous sequence of order type $\omega_{1}$, while it is easy to see that no such sequence exists in $D_{0}$. Hence $D_{0} \cong D_{1}$. If we construct $D_{0}, D_{1}$ in a standard model $\mathrm{M}$ of ZFC we obtain 
(because of the absoluteness of their definition) that $D_{0}$ and $D_{1}$ are not isomorphic in any extension $\mathbf{N}$ of $\mathbf{M}$ for which $\omega_{1}^{\mathbf{N}}=\omega_{1}^{\mathbf{M}}$. This proves Theorem 2.2 for the case $\lambda=\omega_{1}$.

We get more interesting results by using Conway's classification in [Co] of the $\omega_{1}$-like d.l.o.'s. With each set $A \subseteq \omega_{1}$ associate an ordered set $\Phi(A)$ whose order type is $\Sigma_{\alpha<\omega_{1}} \tau_{\alpha}$ where

$$
\tau_{\alpha}=\left\{\begin{array}{ll}
1+\eta & \alpha \in A \\
\eta & \alpha \notin A
\end{array}\right\}
$$

$\Phi(A)$ is always an $\omega_{1}$-like d.l.o., which has a first element iff $0 \in A$. Conway's result is that every $\omega_{1}$-like d.l.o. is isomorphic to $\Phi(A)$ for some $A \subseteq \omega_{1}$, and that if $A, B \subseteq \omega_{1}, 0 \in A, 0 \in B$ then $\Phi(A) \simeq \Phi(B)$ iff $A \sim B$ [where $~$ denotes equivalence with respect to the filter on $\omega_{1}$ generated by the closed unbounded sets, i.e., $A \sim B$ iff $A \cap C=B \cap C$ for some closed unbounded $\left.C \subseteq \omega_{1}\right]$.

Now fix some countable standard model $\mathbf{M}$ of ZFC and work within $\mathbf{M}$. Let $A$ be a stationary subset of $\omega_{1}$ containing 0 whose complement is also stationary (the existence of such sets is proved, e.g., in [Je2, p. 170]). Consider the three orderings

$$
D_{0}=\Phi(\{0\}), \quad D=\Phi(A) \text { and } D_{1}=\Phi\left(\omega_{1}\right) .
$$

Since the three sets $\{0\}, A, \omega_{1}$ are pairwise nonequivalent, the orderings $D_{0}$, $D, D_{1}$ are not isomorphic (in $M$ ). However, we claim that $D_{0}$ and $D$ are $\omega_{1}$-potentially isomorphic over $M$, and so are $D$ and $D_{1}$ (but not $D_{0}$ and $D_{1}$, as noted above).

To prove $D\left(\omega_{1}-\mathrm{PI}\right) D_{1}$ over $\mathbf{M}$ it suffices to show that there is an extension $\mathbf{N}$ of $M$ such that $N \cap^{\omega} M \subseteq M$ and $D \simeq D_{1}$ in $N$. Since the operation $\Phi$ is absolute we need only choose $\mathrm{N}$ so that $N \cap^{\omega} M \subseteq M$ and in $\mathrm{N} A \sim \omega_{1}$, that is, $A$ contains a closed unbounded set. The existence of a Cohen extension that adds no new $\omega$-sequences but adds a closed unbounded subset to a given stationary set $A \subseteq \omega_{1}$ is in fact known, and is the result of Baumgartner, Harrington and Kleinberg [BHK]. Thus $D\left(\omega_{1}-\mathrm{PI}\right) D_{1}$ over $\mathbf{M}$.

The proof that $D_{0}\left(\omega_{1}-\mathrm{PI}\right) D$ over $\mathbf{M}$ is similar. One adds a closed unbounded subset to $\omega_{1}-A$, thereby making $A \sim\{0\}$ and $D \simeq D_{0}$.

Summarizing, we have proved

3.1. THEOREM. Let $\mathbf{M}$ be a countable standard model of $Z F C$. There exist $D_{0}$, $D, D_{1} \in M$ such that

(i) in $M, D_{0}, D$ and $D_{1}$ are three nonisomorphic $L_{\infty \omega_{1}}$-equicalent $\omega_{1}$-like d.l.o.'s;

(ii) $D_{0}\left(\omega_{1}-\mathrm{PI}\right) D$ and $D\left(\omega_{1}-\mathrm{PI}\right) D_{1}$ over $\mathrm{M}$ but $D_{0}$ and $D_{1}$, are not $\omega_{1}-\mathrm{PI}$ over M.

3.2. Corollaries. (a) Two structures of cardinality $\omega_{1}$ in $\mathbf{M}$ which are $\omega_{1}-\mathrm{PI}$ over $\mathbf{M}$ need not be isomorphic $\mathbf{M}$. 
(b) The relation " $\omega_{1}-\mathrm{PI}$ over $\mathbf{M}$ " is not transitive, even when restricted to structures of cardinality $\omega_{1}$.

Problem. Prove or give a counterexample (for structures A, B $\in \mathbf{M}$ with one binary relation, say): If $\mathbf{A} \equiv_{\infty \omega_{1}} B$ in $\mathbf{M}$ then there exists a finite sequence $\mathbf{A}=\mathbf{A}_{0}, \mathbf{A}_{1}, \ldots, \mathbf{A}_{n}=\mathbf{B}$ such that $\mathbf{A}_{k}\left(\omega_{1}-\mathrm{PI}\right) \mathbf{A}_{k+1}$ over $\mathbf{M}$ for each $k<n$.

4. $\kappa^{+}$-like dense linear orderings (the regular case). The proof that $\Phi(A)$ $\equiv_{\infty \omega_{1}} \Phi(B)$ for all $A, B \subseteq \omega_{1}$ such that $0 \in A \cap B$ used very strongly the uniqueness of a countable d.l.o. without first or last element (this goes into verifying that the set $I$ of partial isomorphisms defined at the beginning of $\$ 3$ has the $<\omega_{1}$-back-and-forth property). Therefore the construction of a similar example of $\kappa^{+}$-like d.l.o.'s which are $L_{\infty \kappa^{+}}$-equivalent but not isomorphic requires some care.

4.1. Proposition. For each infinite cardinal $\kappa$ there exists a dense-linearorder-type $\eta$, of cardinality $\kappa$, satisfying $\eta \cdot \kappa=\eta+1+\eta=\eta$ (in forming $\eta \cdot \kappa$ we regard $\kappa$, of course, as an order type).

Proof. First assume GCH. Let $\eta$ be the saturated d.l.o. without endpoints of cardinality $\kappa$ if $\kappa$ is regular or the special d.l.o. of cardinality $\kappa$ if $\kappa$ is singular (see [CK]). For regular $\kappa, \eta$ is characterized as the unique $\kappa$-dense linear order of cardinality $\kappa$ (see [Sa, p. 77]), but it is easy to see that if $\eta$ is $\kappa$-dense so are $\eta \cdot \kappa$ and $\eta+1+\eta$, hence $\eta \cdot \kappa=\eta+1+\eta=\eta$. For singular $\kappa$ one uses a specializing chain for (an ordered set of type) $\eta$ to get specializing chains for $\eta \cdot \kappa$ and for $\eta+1+\eta$ (details are left to the reader). By the uniqueness of special models $\eta \cdot \kappa=\eta+1+\eta=\eta$.

To eliminate the $\mathrm{GCH}$, regard the given cardinal $\kappa$ as a cardinal in the constructible universe $L$ (in which the GCH holds) and find in $L$ an ordered set $H$ of power $\kappa$ whose order-type $\eta$ satisfies (in $L$ ) $\eta \cdot \kappa=\eta+1+\eta=\eta$. Clearly $\eta$ then has the same properties in the real universe $V$.

Now let $\kappa$ be fixed and choose $\eta$ as in Proposition 2.1 (more precisely, fix an ordered set $H$ whose order-type $\eta$ has these properties). With each set $A \subseteq \kappa^{+}$we associate an ordered set $\Phi(A)$ of type $\Sigma_{\alpha<\kappa^{+}} \tau_{\alpha}$ where

$$
\tau_{\alpha}=\left\{\begin{array}{ll}
1+\eta & \alpha \in A \\
\eta & \alpha \notin A
\end{array}\right\} .
$$

Unfortunately, it is not true in general that $\Phi(A) \equiv_{\infty \infty k^{+}} \Phi(B)$ whenever $A$, $B \subseteq \kappa^{+}, 0 \in A \cap B$. [For example, if $\kappa>\omega$ is regular and $\eta$ happens to be the saturated d.l.o. type of cardinality $\kappa$, then the set of predecessors of every element in $\eta$ has cofinality $\kappa$. The same holds for every element of $\Phi(\{0\})$ except the first, but if $A$ contains a limit ordinal of cofinality $\mu<\kappa$ then $\Phi(A)$ contains an element whose set of predecessors has the same cofinality $\mu$. This easily implies $\Phi(\{0\}) Z_{\infty} \Phi(A)$.]

However, we can still prove the following (assuming $\kappa$ and $\eta$ are related as in Proposition 4.1). 
4.2. Proposition. Let $A \subseteq \kappa^{+}, 0 \in A$ and suppose that each limit ordinal $\alpha \in A$ satisfies $\operatorname{cf}(\alpha)=\kappa$. Then $\Phi(A) \equiv_{\infty \kappa^{+}} \Phi(\{0\})$.

This proposition is of interest only if $\kappa$ is regular. We present the proof in some detail in order to save work in $\$ 5$.

To prove 4.2 we shall prove first several properties of $\eta$, where $\kappa, \eta$ are as in 4.1.

LEMMA $1 \cdot \eta \cdot(\alpha+1)=\eta$ for all $\alpha \leqslant \kappa$.

Proof. First assume $\alpha<\kappa$. Then $\eta \cdot(\alpha+1)=\eta \cdot \alpha+\eta=\eta \cdot \alpha+\eta \cdot \kappa$ $=\eta \cdot(\alpha+\kappa)=\eta \cdot \kappa=\eta$. In particular $\eta+\eta=\eta \cdot(1+1)=\eta$. Hence $\eta$. $(\kappa+1)=\eta \cdot \kappa+\eta=\eta+\eta=\eta$.

LEMMA 2. $\eta \cdot \operatorname{cf}(\kappa)=\eta$.

Proof. If $\kappa$ is regular $\eta \cdot \operatorname{cf}(\kappa)=\eta \cdot \kappa=\eta$. Now let $\kappa$ be singular, $\operatorname{cf}(\kappa)=$ $\mu$. Choose an increasing sequence $\left\langle\alpha_{i} \mid i<\mu\right\rangle$ such that $\sup _{i<\mu} \alpha_{i}=\kappa$ and each $\alpha_{i}$ is a successor ordinal. Define $\beta_{j}(j<\mu)$ by: $\beta_{0}=\alpha_{0}, \beta_{i+1}=\alpha_{i+1}-\alpha_{i}$, and for limit ordinals $j<\mu: \beta_{j}=\alpha_{j}-\sup _{i<j} \alpha_{i}$. Thus $\alpha_{0}=\beta_{0}, \alpha_{1}=\beta_{0}+\beta_{1}$ and in general, $\alpha_{j}=\sum_{i<j} \beta_{i}(j<\mu), \sum_{i<\mu} \beta_{i}=\kappa$. Note also that each $\beta_{i}$ is a successor ordinal $<\kappa$. By Lemma $1, \eta \cdot \beta_{i}=\eta$. Thus

$$
\eta=\eta \cdot \kappa=\eta \cdot \sum_{i<\mu} \beta_{i}=\sum_{i<\mu} \eta \cdot \beta_{i}=\sum_{i<\mu} \eta=\eta \cdot \mu .
$$

Lemma 3. Let $A \subseteq \kappa^{+}, 0 \in A$ and define

$$
\tau_{\alpha}=\left\{\begin{array}{ll}
1+\eta & \alpha \in A \\
\eta & \alpha \notin A
\end{array}\right\}
$$

Suppose each limit ordinal $\alpha \in A$ satisfies $\operatorname{cf}(\alpha)=\kappa$. For $\delta<\kappa^{+}$put $\sigma_{\delta}=$ $\Sigma_{\alpha<\delta} \tau_{\alpha}$. Then for each $\delta\left(0<\delta<\kappa^{+}\right) \sigma_{\delta}=1+\eta \cdot \operatorname{cf}(\delta)(=1+\eta$ if $\delta$ is a successor ordinal).

Proof. We proceed by induction on $\delta\left(0<\delta<\kappa^{+}\right)$, using Lemmas 1,2 and the equation $\eta \cdot \kappa=\eta+1+\eta=\eta+\eta=\eta$.

(I) $\delta=1 . \sigma_{\delta}=\tau_{0}=1+\eta=1+\eta \cdot \operatorname{cf}(\delta)$.

(II) $\delta=\gamma+1, \gamma>0$. By induction hypothesis $\sigma_{\gamma}=1+\eta \cdot \operatorname{cf}(\gamma)$. If $\operatorname{cf}(\gamma)$ $=1$ or $\operatorname{cf}(\gamma)=\kappa$ then $\sigma_{\gamma}=1+\eta$ and

$$
\sigma_{\delta}=\sigma_{\gamma}+\tau_{\gamma}=\left\{\begin{array}{ll}
1+\eta+1+\eta & \text { if } \gamma \in A \\
1+\eta+\eta & \text { if } \gamma \notin A
\end{array}\right\}=1+\eta=1+\eta \cdot \operatorname{cf}(\delta) .
$$

If $1<\operatorname{cf}(\gamma)<\kappa$ then by the hypothesis on $A, \gamma \notin A$ so $\tau_{\gamma}=\eta$. Thus

$$
\begin{aligned}
\sigma_{\delta} & =\sigma_{\gamma}+\tau_{\gamma}=1+\eta \cdot \operatorname{cf}(\gamma)+\eta \\
& =1+\eta(\operatorname{cf}(\gamma)+1)=1+\eta=1+\eta \cdot \operatorname{cf}(\delta) .
\end{aligned}
$$

(III) $\operatorname{cf}(\delta)=\omega$. Choose an increasing sequence $\left\langle\alpha_{n}\right| n\langle\omega\rangle$ of successor ordinals such that $\delta=\sup _{n<\omega} \alpha_{n}$. Let 


$$
\begin{aligned}
\mu_{0} & =\sum_{\alpha<\alpha_{0}} \tau_{\alpha}, \\
\mu_{n+1} & =\sum_{\alpha_{n}<\alpha<\alpha_{n+1}} \tau_{\alpha} \quad(n<\omega) .
\end{aligned}
$$

Thus $\sigma_{\delta}=\Sigma_{n<\omega} \mu_{n}$. Each $\mu_{n}$ is a sum of $\eta$ 's and $1+\eta$ 's, that is, we may write

$$
\mu_{n}=\sum_{\beta<\beta_{n}} \tau_{\beta}^{(n)} \text { where } \beta_{0}=\alpha_{0}, \beta_{n+1}=\alpha_{n+1}-\alpha_{n}
$$

and

$$
\tau_{\beta}^{(n)}=\left\{\begin{array}{ll}
1+\eta & \text { if } \alpha_{n-1}+\beta \in A \\
\eta & \text { if } \alpha_{n-1}+\beta \notin A
\end{array}\right\}
$$

(Letting $\alpha_{-1}=0$ ). Thus the $\mu_{n}$ 's themselves are sums of the form to which we may apply our induction hypothesis, with the "shifted" set $A^{(n)}(=\{\alpha-$ $\left.\alpha_{n-1} \mid \alpha \in A, \alpha_{n-1}<\alpha<\alpha_{n}\right\}$ ) playing the role of $A$. Note that $A^{(n)}$ does not contain limit ordinals of cofinality $\neq \kappa$, but it need not contain 0 . Applying the induction hypothesis to $A^{(n)}, \beta_{n}$ (and adding 0 to $A_{n}$ if needed) we see that

$$
\mu_{n}=1+\eta \cdot \operatorname{cf}\left(\beta_{n}\right)=1+\eta \quad \text { if } 0 \in A^{(n)}
$$

and

$$
\mu_{n}=\eta \cdot \operatorname{cf}\left(\beta_{n}\right)=\eta \quad \text { if } 0 \notin A^{(n)} .
$$

Since $0 \in A^{(0)}$ we see that $\mu_{0}=1+\eta$ and $\sigma_{8}=1+\eta+\mu_{1}+\mu_{2}+\ldots$ where each $\mu_{\eta}$ is $\eta$ or $1+\eta$. It is easy to see that the l's can be absorbed so that $\sigma_{\delta}=1+\eta \cdot \omega=1+\eta \cdot \operatorname{cf}(\delta)$.

(IV) $\operatorname{cf}(\delta)>\omega$. By choosing first some increasing sequence of order type $\operatorname{cf}(\delta)$ which tends to $\delta$ and then taking its limit points we get an increasing continuous sequence $\left\langle\alpha_{i} \mid i<\operatorname{cf}(\delta)\right\rangle$ such that $\delta=\sup _{i<\mathrm{cf}(\delta)} \alpha_{i}$ and for each $i$, $\omega<\operatorname{cf}\left(\alpha_{i}\right)<\operatorname{cf}(\delta)$ hence $\alpha_{i} \notin A$. We can now write $\sigma_{\delta}$ in the form

$$
\begin{aligned}
\sigma_{\delta}= & \sum_{\alpha<\alpha_{0}} \tau_{\alpha}+\tau_{\alpha_{0}}+\sum_{\alpha_{0}<\alpha<\alpha_{1}} \tau_{\alpha}+\tau_{\alpha_{1}}+\cdots+\tau_{\alpha_{\omega}} \\
& +\sum_{\alpha_{\omega}<\alpha<\alpha_{\omega+1}} \tau_{\alpha}+\tau_{\alpha_{\omega+1}}+\cdots
\end{aligned}
$$

where each $\tau_{\alpha_{i}}$ is $\eta$ since $\alpha_{i} \notin A$. As for sums of the form $\Sigma_{\alpha_{1}<\alpha<\alpha_{i+1}} \tau_{\alpha}$, the induction hypothesis implies (by an argument similar to that of case (III)) that it is $1+\eta \cdot \operatorname{cf}\left(\alpha_{i+1}\right)$ or $\eta \cdot \operatorname{cf}\left(\alpha_{i+1}\right)$ (according as $\alpha_{i}+1$ is in $A$ or not in $A$ ). Similarly $\Sigma_{\alpha<\alpha_{0} \tau_{\alpha}}=1+\eta$. We now use the generalized associative law and the fact that $\tau_{\alpha_{i}}=\eta=\eta+\eta$ to write our sum in the form: 


$$
\begin{aligned}
\sigma_{\delta}= & (1+\eta)+\left(\eta+\sum_{\alpha_{0}<\alpha<\alpha_{1}} \tau_{\alpha}+\eta\right)+\left(\eta+\sum_{\alpha_{1}<\alpha<\alpha_{2}} \tau_{\alpha}+\eta\right) \\
& +\cdots+\left(\eta+\sum_{\alpha_{\omega}<\alpha<\alpha_{\omega+1}} \tau_{\alpha}+\eta\right)+\cdots \\
= & 1+\eta+\sum_{i<\operatorname{cf}(\delta)}\left(\eta+\sum_{\alpha_{i}<\alpha<\alpha_{i+1}} \tau_{\alpha}+\eta\right) .
\end{aligned}
$$

But

$$
\sum_{\alpha_{i}<\alpha<\alpha_{i+1}} \tau_{\alpha}+\eta=\left\{\begin{array}{ll}
1+\eta \cdot\left(\operatorname{cf}\left(\alpha_{i+1}\right)+1\right)=1+\eta & \text { if } \alpha_{i}+1 \in A \\
\eta \cdot\left(\operatorname{cf}\left(\alpha_{i+1}\right)+1\right)=\eta & \text { if } \alpha_{i}+1 \notin A
\end{array}\right\}
$$

and $\eta+1+\eta=\eta+\eta=\eta$. Thus

$$
\sigma_{\delta}=1+\eta+\eta \cdot \operatorname{cf}(\delta)=1+\eta(1+\operatorname{cf}(\delta))=1+\eta \cdot \operatorname{cf}(\delta) .
$$

We are now ready for the proof of Proposition 4.2. Let $A$ be a set as assumed there. Regard $\Phi(A)$ as consisting of $\kappa^{+}$blocks, the $\alpha$ th block $B_{\alpha}$ having order type $\tau_{\alpha}$. By a good cut in $\Phi(A)$ we mean an initial segment $S$ of $\Phi(A)$ which "cuts some block in the middle", i.e.-for some unique $\alpha$ the order type of $B_{\alpha}-S$ is $\eta$ and the order type of $B_{\alpha} \cap S$ is the same as that of $B_{\alpha}$. Since $\eta+\eta=\eta$ we have $\tau_{\alpha}+\eta=\tau_{\alpha}$ and so for every $\alpha<\kappa^{+}$there is a good cut which cuts $B_{\alpha}$ in the middle. It follows from Lemma 3 (cf. the last two sentences in the proof of Lemma 3 for a similar argument) that if $S_{1}$ and $S_{2}$ are good cuts in $\Phi(A)$ and $S_{1} \subsetneq S_{2}$ then the order type of $S_{2}-S_{1}$ is exactly $\eta$, except perhaps when they cut the same block.

The notion of a good cut and the remarks just made about good cuts are clearly applicable to $\Phi(\{0\})$ as well. Now let $I=\{f \mid f$ is a partial isomorphism from $\Phi(\{0\})$ to $\Phi(A), \operatorname{dom}(f)$ is a good cut in $\Phi(\{0\})$ and range $(f)$ is a good cut in $\Phi(A)\}$. It is almost immediate from the above remarks that $I: \Phi(\{0\}) \cong_{\kappa^{+}} \Phi(A)$, hence $\Phi(\{0\}) \equiv_{\infty k^{+}} \Phi(A)$.

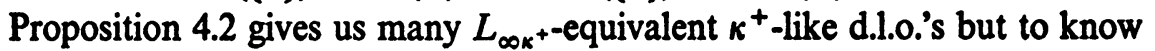
that they are not isomorphic we have to extend Conway's result from $\omega_{1}$ to $\kappa^{+}$. Recall that $\kappa$ and $\eta$ are fixed and supposed to satisfy 4.1.

4.3. Proposition. Let $A, B \subseteq \kappa^{+}, 0 \in A, 0 \in B$. Suppose each limit $\alpha \in A$ $\cup B$ satisfies $\operatorname{cf}(\alpha)=\kappa$. Then $\Phi(A) \simeq \Phi(B)$ iff $A \cap C=B \cap C$ for some closed unbounded set $C \subseteq \kappa^{+}$.

(We need only one direction but for completeness prove both.)

Proof. $\Rightarrow$. Assume $\Phi(A) \simeq \Phi(B)$. Let $D$ and $D^{\prime}$ be the Dedekind completions of $\Phi(A), \Phi(B)$ respectively. For each $\alpha<\kappa^{+}$let $d_{\alpha}\left(d_{\alpha}^{\prime}\right)$ be the inf in $D\left(D^{\prime}\right.$ resp.) of the $\alpha$ th block of $\Phi(A)$ (of $\Phi(B)$ resp.). Thus $d_{\alpha} \in \Phi(A)$ if $\alpha \in A, d_{\alpha}^{\prime} \in \Phi(B)$ iff $\alpha \in B .\left\langle d_{\alpha} \mid \alpha<\kappa^{+}\right\rangle$and $\left\langle d_{\alpha}^{\prime}\right| \alpha\left\langle\kappa^{+}\right\rangle$are increasing continuous cofinal sequences in $D, D^{\prime}$ resp. 
Since $\Phi(A) \cong \Phi(B)$ there is an isomorphism $h$ of $D$ and $D^{\prime}$ which maps $\Phi(A)$ onto $\Phi(B)$. Then $\left\langle h\left(d_{\alpha}\right)\right| \alpha\left\langle\kappa^{+}\right\rangle$and $\left\langle d_{\alpha}^{\prime}\right| \alpha\left\langle\kappa^{+}\right\rangle$are two increasing continuous cofinal sequences in $D^{\prime}$. Since $\left.\operatorname{cf}\left(D^{\prime}\right)=\kappa^{+}\right\rangle \omega$ it follows that the set $C=\left\{\alpha<\kappa^{+} \mid h\left(d_{\alpha}\right)=d_{\alpha}^{\prime}\right\}$ is closed unbounded in $\kappa^{+}$(cf. the proof that the intersection of two closed unbounded sets is unbounded). For $\alpha \in C$ we have $\alpha \in A \Leftrightarrow d_{\alpha} \in \Phi(A) \Leftrightarrow d_{\alpha}^{\prime} \in \Phi(B) \Leftrightarrow \alpha \in B$. Thus $A \cap C=B \cap C$.

$\Leftarrow$. Suppose $A \cap C=B \cap C$ for some closed unbounded set $C \subseteq \kappa^{+}$. Since $0 \in A \cap B$ we may assume $0 \in C$. Let $\left\langle\gamma_{i}\right| i\left\langle\kappa^{+}\right\rangle$be the increasing enumeration of $C\left(\gamma_{0}=0\right)$. Let, as usual

$$
\tau_{\alpha}=\left\{\begin{array}{ll}
1+\eta & \text { if } \alpha \in A \\
\eta & \text { if } \alpha \notin A
\end{array}\right\} \quad\left(\alpha<\kappa^{+}\right) .
$$

The order type of $\Phi(A)$ is

$$
\sum_{\alpha<\kappa^{+}} \tau_{\alpha}=\sum_{i<\kappa^{+}} \sum_{\gamma_{i}<\alpha<\gamma_{i+1}} \tau_{\alpha},
$$

where by Lemma $3 \Sigma_{\gamma_{i}<\alpha<\gamma_{i+1}} \tau_{\alpha}$ is $1+\eta \cdot \operatorname{cf}\left(\gamma_{i+1}\right)$ if $\gamma_{i} \in A, \eta \cdot \operatorname{cf}\left(\gamma_{i+1}\right)$ if $\gamma_{i} \notin A$. Thus the order type of $\Phi(A)$ is $\Sigma_{i<\kappa^{+}} \mu_{i}$ where

$$
\mu_{i}= \begin{cases}1+\eta \cdot \operatorname{cf}\left(\gamma_{i+1}\right) & \text { if } \gamma_{i} \in A, \\ \eta \cdot \operatorname{cf}\left(\gamma_{i+1}\right) & \text { if } \gamma_{i} \notin A .\end{cases}
$$

It is clear that the order type of $\Phi(B)$ is exactly the same since a similar argument applies to $B$ and $\gamma_{i} \in A \Leftrightarrow \gamma_{i} \in B$ for each $i$. Thus $\Phi(A) \simeq \Phi(B)$.

We can now combine 4.2 and 4.3 to get some examples. Assume that $\kappa$ is regular and let

$$
A_{1}=\left\{\delta<\kappa^{+} \mid \operatorname{cf}(\delta)=\kappa\right\} \cup\{0\} .
$$

Put $D_{0}=\Phi(\{0\})$ and $D_{1}=\Phi\left(A_{1}\right) \cdot A_{1}$ is clearly stationary so by $4.3, D_{0} \approx D_{1}$ while by $4.2, D_{0} \equiv_{\infty \kappa^{+}} D_{1}$.

Note that the orderings $D_{0}, D_{1}$ have been defined in an explicit way from $\eta$. $\kappa^{+}$and $\left\{\delta<\kappa^{+} \mid \operatorname{cf}(\delta)=\kappa\right\}$, and if $\eta$ satisfies 4.1 in $M$ it satisfies 4.1 in every $\mathbf{N} \supseteq \mathbf{M}$. Thus if we construct $D_{0}, D_{1}$ in a standard model $\mathbf{M}(\kappa$ being a regular cardinal of $\mathbf{M}$ ) they will remain nonisomorphic in any $\mathbf{N} \supseteq \mathbf{M}$ where $\kappa$ remains regular (so that $\mathrm{cf}^{\mathrm{M}}(\delta)=\kappa \Rightarrow \mathrm{cf}^{\mathrm{N}}(\delta)=\kappa$ ) and $\left(\kappa^{+}\right)^{\mathrm{M}}=\left(\kappa^{+}\right)^{\mathrm{N}}$. This proves Theorem 2.2.

To get the nontransitivity result of $\S 3$ we have to work a bit harder. Let $\mathbf{M}$ be a countable standard model of ZFC, $\kappa$ a regular cardinal in $M, \eta$ an order type in $M$ satisfying 4.1 and define $D_{0}=\Phi(\{0\}), D_{1}=\Phi\left(A_{1}\right)$ as before. We have just seen that $D_{0}$ and $D_{1}$ are not $\kappa^{+}$-PI over M. Can we find $A \subseteq \kappa^{+}$in $M$ such that each of $D_{0}, D_{1}$ is $\kappa^{+}$-PI over $\mathrm{M}$ to $D=\Phi(A)$ ?

By 4.2 it would suffice to find $A \in M$ with the following properties:

(1) In M: $A \subseteq \kappa^{+}, 0 \in A$ and each limit ordinal in $A$ has cofinality $\kappa$. 
(2) There is an extension $\mathrm{N}_{0}$ of $\mathrm{M}$ such that $N_{0} \cap^{\kappa} M \subseteq M$ and in $\mathrm{N}_{0}, A$ is nonstationary (so that $\Phi(A) \simeq \Phi(\{0\})$ ).

(3) There is an extension $\mathrm{N}_{1}$ of $\mathbf{M}$ such that $N_{1} \cap^{\kappa} M \subseteq M$ and in $\mathrm{N}_{1}$, $A_{1}-A$ is nonstationary (so that $\left.\Phi(A) \cong \Phi\left(A_{1}\right)\right)$. $\left[A-A_{1}\right.$ is obviously nonstationary, as it contains (by (1)) only successor ordinals; in fact we may assume $A \subseteq A_{1}$.]

In other words, the problem is to divide the set $\left\{\delta<\kappa^{+} \mid \operatorname{cf}(\delta)=\kappa\right\}$ into two disjoint parts each of which can be made nonstationary in a suitable (Cohen) extension of $M$ with no new sequences of length $\kappa$.

For $\kappa=\omega$ we solved this problem by using the result of [BHK]. For $\kappa>\omega$ one has to generalize the result of [BHK] to higher cardinals in a suitable way. This has been done explicitly in [St1] so as to give a positive solution to the above problem under the assumption that $\kappa=\kappa^{\mathrm{s}}$ in $\mathbf{M}$. The methods of proof in [St1] overlap those of the earlier work of Gregory-Harrington [GH] so that the result is essentially already in [GH].

Assuming this result of [GH] and [St1] we conclude.

4.4. Theorem. Let $M$ be a countable standard model of $Z F C, \kappa, \lambda$ infinite cardinals of $\mathbf{M}$ such that $\kappa=\psi^{\psi}, \lambda=\kappa^{+}$(in $\left.\mathbf{M}\right)$. Then there exist $D_{0}, D$, $D_{1} \in M$ satisfying the conclusions of Theorem 3.1 with $\omega_{1}$ replaced by $\lambda$. Hence Corollaries 3.2 hold with $\omega_{1}$ replaced by $\lambda$.

5. $\mathrm{K}^{+}$-like dense linear orderings (the singular case). Proposition 4.2 is not of much use when $\kappa$ is singular for then it applies only to sets $A \subseteq \kappa^{+}$that contain no limit ordinals. For such a set $A \Phi(A)$ is isomorphic to $\Phi(\{0\})$ by Proposition 4.3.

Now suppose we replace in the hypotheses on 4.2 the equation " $\operatorname{cf}(\alpha)=\kappa$ " by "cf( $\alpha)=\operatorname{cf}(\kappa)$ ". Is the resulting statement true? We shall see below (5.5) that it is not, at least when $\eta$ is the special d.l.o. of cardinality $\kappa$ (which exists if the GCH holds). However, a surprisingly large part of the proof of 4.2 remains valid. Lemmas 1 and 2 certainly hold (they do not mention the set $A$ ). In the proof of Lemma 3 one can do cases (I), (II), (III) as before (with some trivial changes in case (II)). The only essential use of the assumption that each limit ordinal in $A$ has cofinality $\kappa$ (rather than cofinality $\operatorname{cf}(\kappa)$ ) is in the first few lines of case (IV) where we establish the following:

(*) If $\delta<\kappa^{+}, \operatorname{cf}(\delta)>\omega$, then there is an increasing continuous sequence $\left\langle\alpha_{i}\right| i\langle\operatorname{cf}(\delta)\rangle$ such that

$$
\delta=\sup _{i<\mathrm{cf}(\delta)} \alpha_{i} \text { and } \alpha_{i} \notin A \text { for each } i .
$$

Once this is established the rest of the proof goes through. However, if $\kappa$ is singular and we take, say,

$$
A=\left\{\alpha<\kappa^{+} \mid \operatorname{cf}(\alpha)=\operatorname{cf}(\kappa)\right\} \cup\{0\} \text { and } \delta=(\operatorname{cf}(\kappa))^{+}
$$

it is clear that (*) fails since $A \cap \delta$ is stationary in $\delta$. Thus we need (*) as an extra hypothesis about $A$. Note that (*) is equivalent to saying that $\delta<\kappa^{+}$, 
$\operatorname{cf}(\delta)>\omega \Rightarrow A \cap \delta$ is nonstationary in $\delta$.

Our argument thus suggests the following generalization of 4.2 (which is of interest mainly if $\kappa$ is singular). We are continuing to assume $\eta$ and $\kappa$ are related as in Proposition 4.1.

5.1. TheOREM. Let $A \subseteq \kappa^{+}, 0 \in A$ and suppose that

(i) for each limit ordinal $\alpha \in A, \operatorname{cf}(\alpha)=\operatorname{cf}(\kappa)$;

(ii) for each $\delta<\kappa^{+}$, if $\operatorname{cf}(\delta)>\omega$ then $A \cap \delta$ is nonstationary in $\delta$.

Then $\Phi(A) \equiv_{\infty \kappa^{+}} \Phi(\{0\})$.

Though the argument above is not a full proof the reader will have no difficulty in convincing himself that the proof of 4.2 actually establishes 5.1. In the same way, the proof of 4.3 immediately generalizes to give

5.2. Theorem. Let $A, B \subseteq \kappa^{+}, 0 \in A, 0 \in B$. Suppose that

(i) each limit ordinal $\alpha \in A \cup B$ satisfies $\operatorname{cf}(\alpha)=\operatorname{cf}(\kappa)$;

(ii) for each $\delta<\kappa^{+}$, if $\operatorname{cf}(\delta)>\omega$ then both $A \cap \delta$ and $B \cap \delta$ are nonstationary in $\delta$.

Then $\Phi(A) \cong \Phi(B)$ iff $A \cap C=B \cap C$ for some closed unbounded $C \subseteq$ $\kappa^{+}$.

[Note that the proof of the $\Rightarrow$ direction in 4.3 applies to arbitrary $A$, $B \subseteq \kappa^{+}$. It is only in the $\Leftarrow$ direction that the assumptions on $A, B$ are used at all.]

Combining 5.1 and 5.2 we get

5.3. Corollary. If $A \subseteq \kappa^{+}, 0 \in A$, each limit ordinal in $A$ has cofinality $\operatorname{cf}(\kappa), A$ is stationary in $\kappa^{+}$but $A \cap \delta$ is nonstationary in any $\delta<\kappa^{+}$of

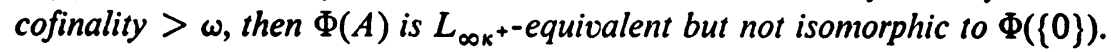

We are left with the problem of finding such a set $A$ (note incidentally that if $A$ is a set as required here and $A_{1}=\{\alpha \in A \mid \alpha=0$ or $\operatorname{Lim}(\alpha)\}$ then $A_{1}$ has all the required properties). For regular $\kappa$ we took $A=\left\{\alpha<\kappa^{+} \mid \operatorname{cf}(\alpha)=\kappa\right\}$ $\cup\{0\}$, but we are now interested in singular $\kappa$. Recall the principle $\square_{\kappa}$ of Jensen [Jn, p. 282]:

$\left(\square_{\kappa}\right)$. There is a sequence $\left\langle C_{\delta} \mid \delta<\kappa^{+}, \operatorname{Lim}(\delta)\right\rangle$ such that

(i) $C_{\delta}$ is closed, unbounded in $\delta$;

(ii) if $\operatorname{cf}(\delta)<\kappa$ then $\left|C_{\delta}\right|<\kappa$;

(iii) if $\gamma$ is a limit point of $C_{\delta}$, then $C_{\gamma}=\gamma \cap C_{\delta}$.

Jensen proved [Jn, Theorem 5.2] that if $V=L$ then $\square_{\kappa}$ holds for each infinite cardinal $\kappa$. The following lemma was proved by M. Magidor. It is of interest even for regular $\kappa$.

5.4. Lemma. Assume $\square_{\kappa}$ and let $\mu$ be a regular cardinal $\leqslant \kappa$. Then there is a set $B \subseteq\left\{\alpha<\kappa^{+} \mid \operatorname{cf}(\alpha)=\mu\right\}$ such that $B$ is stationary on $\kappa^{+}$but $B \cap \delta$ is nonstationary on $\delta$ for each limit ordinal $\delta<\kappa^{+}$. [If $\mu=\kappa$ take $B=\{\alpha<$ $\left.\kappa^{+} \mid \operatorname{cf}(\alpha)=\kappa\right\}$; for this case $\square_{\kappa}$ is not needed.] 
Proof. Let $\left\langle C_{\delta}\right| \delta\left\langle\kappa^{+}, \operatorname{Lim}(\delta)\right\rangle$ be a sequence as required in $\square_{\kappa^{\text {. }}}$. It follows from (ii), (iii) of $\square_{\kappa}$ that for each $\delta$ the order type of $C_{\delta}$ is $<\kappa$. For each $\alpha<\kappa$ put $B_{\alpha}=\left\{\delta<\kappa^{+} \mid \operatorname{cf}(\delta)=\mu\right.$ and the order type of $C_{\delta}$ is exactly $\alpha\}$. Thus $\left\{\delta<\kappa^{+} \mid \operatorname{cf}(\delta)=\mu\right\}=\cup_{\alpha<\kappa} B_{\alpha}$. The left-hand side of this equation is a stationary set on $\kappa^{+}$, hence $B_{\alpha}$ is stationary for at least one $\alpha<x$. Choose $\alpha_{0}$ so that $B_{\alpha_{0}}$ is stationary and put $B=B_{\alpha_{0}}$.

Now let $\delta$ be any limit ordinal $<\kappa^{q}$. If $\operatorname{cf}(\delta)=\omega$ there is a closed unbounded set in $\delta$ containing only successor ordinals so $B \cap \delta$ is nonstationary on $\delta$. If $\operatorname{cf}(\delta)>\omega$ then the set $C_{\delta}^{\prime}$ of limit points of $C_{\delta}$ is closed unbounded in $\delta$ while its intersection with $B$ contains at most one point (for if $\xi \in C_{\delta}^{\prime} \cap B$ then $C_{\xi}=\xi \cap C_{\delta}$ and $C_{\xi}$ has order type $\alpha_{0}$ so $\xi$ is the $\alpha_{0}$ th element of $C_{\delta}$ ). So $B \cap \delta$ is again nonstationary on $\delta$.

Clearly if $B$ is as in 5.4 with $\mu=\operatorname{cf}(\kappa)$ and $A=B \cup\{0\}$ then $A$ satisfies the assumptions of 5.3. We have thus proved the following.

5.5. Throrem. Let $\kappa$ be singular and assume $\square_{k}$. Then there exist two

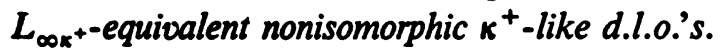

It is natural to ask whether $\Phi(\{0\})$ and $\Phi(A)$, for the set $A=B \cup\{0\}$ just considered, are $\kappa^{+}$-potentially isomorphic. The answer may depend on the choice of the set $B$ satisfying the conclusion of 5.4. We can show that it is possible to choose $B$ in such a way that $\Phi(\{0\})\left(\kappa^{+}-\mathrm{PI}\right) \Phi(A)$. This depends on a refinement of the proof of 5.4-see [St1] for more details. Using this we get the following refinement of 5.5 .

5.6. Proposition. If $\kappa$ is singular and $\square_{\kappa}$ holds, then there exist two $\kappa^{+}$-potentially-isomorphic, nonisomorphic, $\kappa^{+}$-like d.l.o.'s.

5.7. Corollary. Assume $V=L$. Then for every $\kappa$ there exist two $\kappa^{+}$potentially-isomorphic, nonisomorphic, $\kappa^{+}$-like d.l.o.'s.

Proof. Use 4.4 for regular $\kappa$ and 5.6 for singular $\kappa$.

Question. Can the assumption of $\square_{\kappa}$ be dropped from 5.5?

For completeness we conclude this section with a lemma showing that our work in this section was necessary because Proposition 4.2 does indeed become false if we merely replace " $\operatorname{cf}(\alpha)=\kappa$ " by $" \operatorname{cf}(\alpha)=\operatorname{cf}(\kappa)$ " in its statement, assuming $\eta$ is the special d.l.o. of singular cardinality $k$. For example, Lemma 5.8 will immediately imply that for this $\eta \Phi(A) z_{\infty x^{+}} \Phi(\{0\})$ where $A=\left\{\alpha<\kappa^{+} \mid \operatorname{cf}(\alpha)=\operatorname{cf}(\kappa)\right\} \cup\{0\}$. Lemma 5.8 is also interesting in its own right as a converse of Theorem 5.1.

5.8. LEMMA. Let $\eta$ be the order type of a special d.l.o. without endpoints of cardinality $\kappa$ ( $\kappa$ may be regular or singular) and let $A \subseteq \kappa^{+}$. If $\Phi(A)$ $\equiv_{\infty \times}+\Phi(\{0\})$ then

(i) $0 \in A$ and each limit ordinal $\alpha \in A$ satisfies $\operatorname{cf}(\alpha)=\operatorname{cf}(\kappa)$;

(ii) for each limit ordinal $\delta<\kappa^{+}$of cofinality greater than $\omega, A \cap \delta$ is nonstationary on $\delta$. 
Proof (HINTED). (i) is left to the reader. If $\kappa$ is regular (ii) follows from (i) so assume $\kappa$ is singular and let $H$ be a special d.l.o. of cardinality $\kappa$ ( $\eta$ is the order type of $H$ ). The main fact, which is obtained by considering a specializing chain for $H$, is the following, in which $\bar{H}$ denotes the Dedekind completion of $H$.

Fact. Let $\operatorname{cf}(\kappa)\left\langle\lambda\left\langle\kappa, \lambda\right.\right.$ regular, and let $\left\langle a_{i}\right| i\langle\lambda\rangle$ be an increasing continuous sequence in $\bar{H}$. Then $\left\{i<\lambda \mid a_{i} \in H\right\}$ is nonstationary on $\lambda$.

This fact easily implies (ii) for the case $\operatorname{cf}(\delta)>\operatorname{cf}(\kappa)$ while in case $\omega<$ $\operatorname{cf}(\delta)<\operatorname{cf}(\kappa)$ (ii) follows from (i).

6. More on orderings and Boolean algebras. We have seen that, assuming $V=L$, for each successor cardinal $\lambda$ there exist two $L_{\infty \lambda}$-equivalent nonisomorphic $\lambda$-like d.l.o.'s. What about limit cardinals? The following problem is apparently still open $\left({ }^{3}\right)$.

Problem. Let $\lambda$ be a limit cardinal of cofinality $>\omega$. Are there two $L_{\infty \lambda}$-equivalent nonisomorphic linear orderings of cardinality $\lambda$ ? (Assume, if you wish, that $V=L$ holds and $\lambda$ is inaccessible-still we do not know the answer.)

It might be possible to construct an example by generalizing the construction of Paris [Pa] from $\omega_{1}$ to arbitrary regular $\lambda$. We have not done this.

We give here without proof two simple results which may be relevant.

6.1. Proposition. Let $\lambda$ be a limit cardinal. Any two $\lambda$-like $L_{\infty \lambda}$-equivalent linear orderings are isomorphic.

(The easy proof proceeds by classifying the elements according to the cardinality of the set of predecessors.)

6.2. TheOREM. Let A, B be two linear orderings in which no dense orderings of cardinality $\lambda$ can be embedded. If $\mathbf{A} \equiv_{\infty} \mathbf{B}$ then $\mathbf{A} \approx \mathbf{B}$.

For $\lambda=\omega$ this is proved in [Na2, Theorem 4.7] and the extension to arbitrary $\lambda$ makes use of this special case and is not very difficult.

We can use our examples of linear orderings or trees to construct Boolean algebras. There are several distinct ways of associating a Boolean algebra with a linear or partial ordering. We can use one of them, together with Morley's trees example (end of $\S 1$ ), to prove the following theorem (recall the meaning of $N_{\lambda}(\mathbf{A}, \mathbf{B})$ from $\left.\S 1\right)$.

6.3. THEOREM. For every regular uncountable cardinal $\lambda$ there exist two atomic Boolean algebras A, B such that $N_{\lambda}(\mathbf{A}, \mathbf{B})$.

We shall only sketch the proof. For more details see [St2] where it is also shown how to get atomless Boolean algebras $\mathrm{A}, \mathrm{B}$ such that $N_{\lambda}(\mathrm{A}, \mathrm{B})$, as well

(3) It is no longer-see notes added to 88 . 
as such Boolean algebras satisfying some completeness and distributivity conditions (for inaccessible $\lambda$ ).

With each tree $T$ we associate a set-Boolean-algebra $\mathbf{B}_{T}=\left(B_{T}, \cup, \cap, \sim\right.$, $\varnothing, T)\left(\sim b=T-b\right.$ for $\left.b \in B_{T}\right)$. Here $B_{T}$ is the field of subsets of $T$ generated by the sets $P_{t}=\{s \mid s<t\}$ (all $t \in T$ ). A simple application of the back and forth criterion (Theorem 1.1) shows that if $T_{1}$ and $T_{2}$ are $L_{\infty} \lambda^{-}$ equivalent trees then $\mathbf{B}_{T_{1}} \equiv_{\infty \lambda} \mathbf{B}_{T_{2}}$. Clearly also $\left|B_{T}\right|=|T|$ if $T$ is infinite.

Now let $T_{1}$ and $T_{2}$ be the trees in Morley's example. Then $\mathbf{B}_{T_{1}} \equiv_{\infty} \mathbf{B}_{T_{2}}$, $\left|B_{T_{1}}\right|=\left|B_{T_{2}}\right|=\lambda$. It remains to prove that $\mathbf{B}_{T_{1}} \approx \mathbf{B}_{T_{2}}$. Recall that $T_{2}$ has a branch of length $\lambda$ while $T_{1}$ does not. Clearly then $B_{T_{2}}$ has a strictly increasing sequence of length $\lambda$. The main part of the proof is to show that $\mathbf{B}_{T_{1}}$ has no strictly increasing sequence of length $\lambda$. This requires a combinatorial argument whose details are given in [St2]. One uses only the fact that $T_{1}$ is a tree of height $\lambda$ with no branch of length $\lambda$. The particular way in which $T_{1}$ was constructed is not relevant to the proof.

Another way to obtain Boolean algebras is from linear orderings. If $\mathbf{O}=(0,<)$ is a linearly ordered set let $\mathbf{B}_{\mathbf{O}}=\left(B_{0}, \cup, \cap, \sim, \varnothing, O\right)$ $(\sim b=O-b)$ be the Boolean algebra of subsets of $O$ generated by the sets $\{b \mid b<a\}(a \in O)$. We shall call $B_{\mathbf{O}}$ the (half-open) interval algebra of $\mathbf{O}$, for if we assume $O$ has a first element then the elements of $B_{0}$ are just the finite unions of half-open intervals $[a, b)$, for $a<b$ or $[a, \infty)=(=\{x \mid a<$ $x\})$ in 0 . In $\$ \$ 4-5$ we got two $\kappa^{+}$-like orderings $O_{1}, O_{2}$ such that $O_{1}$ $\equiv_{\infty \kappa^{+}} \mathrm{O}_{2}, \mathbf{O}_{1} \approx \mathrm{O}_{2}$. Moreover, for the case $\kappa=\kappa^{\mathrm{s}}$ we got three such orderings that showed the nontransitivity of the relation " $k^{+}-$PI over M" (Theorem 4.4). Let $D_{0}, D$ and $D_{1}$ be the $\kappa^{+}$-like orderings of Theorem 4.4 and let $B_{0,} B$ and $B_{1}$ be the associated interval algebras. A simple back-and-forth argument shows that if two orderings are $L_{\infty \lambda}$-equivalent so are their interval algebras. Hence (in $M$ ) $B_{0}, B$ and $B_{1}$ are $L_{\infty \lambda^{-}}$-equivalent (where $\lambda=\kappa^{+}$). Also, from the absolute way in which $D_{0}, D_{1}$ (hence $\mathbf{B}_{0}, \mathbf{B}_{1}$ ) were defined it follows that if we can prove (in $Z F C$ ) that $B_{0}$ and $B_{1}$ are not isomorphic, then $B_{0}$ and $B_{1}$ are not $\lambda$-PI over $\mathbf{M}$. On the other hand, if $\mathbf{N}$ is an extension of $\mathbf{M}$ in which $D_{0} \cong D$ then clearly, in $\mathbf{N}, \mathbf{B}_{0} \cong \mathbf{B}$. Thus $\mathbf{B}_{0}(\lambda-\mathrm{PI}) \mathbf{B}$ over $\mathbf{M}$ and similarly $\mathbf{B}(\lambda-\mathrm{PI}) \mathbf{B}_{1}$ over $\mathbf{M}$.

Thus, once we prove the nonisomorphism of $\mathbf{B}_{\mathbf{0}}$ and $\mathbf{B}_{1}$ (the interval algebras of $D_{0}=\Phi(\{0\})$ and $D_{1}=\Phi\left(\left\{\delta<\kappa^{+} \mid \operatorname{cf}(\delta)=\kappa\right\} \cup\{0\}\right)$ as defined in $\S 4$ ) we shall establish the following theorem (modulo the facts from [GH] or [St1] stated without proof in $\S 4$ ).

6.4. THEOREM. Let $M$ be a countable standard model of $Z F C, \kappa, \lambda$ infinite cardinals of $\mathbf{M}$ such that in $\mathbf{M} \kappa=\kappa^{5}, \lambda=\kappa^{+}$. There exist (atomic) Boolean algebras $\mathbf{B}_{0}, \mathbf{B}, \mathbf{B}_{1} \in M$ such that in $\mathbf{M}, \mathbf{B}_{0}, \mathbf{B}, \mathbf{B}_{1}$ are pairwise $L_{\infty \lambda}$-equivalent and nonisomorphic, $\mathbf{B}_{0}(\lambda-\mathrm{PI}) \mathbf{B}$ and $\mathbf{B}(\lambda-\mathrm{PI}) \mathbf{B}_{1}$ but not $\mathbf{B}_{0}(\lambda-\mathrm{PI}) \mathbf{B}_{1}$ over $\mathbf{M}$.

[It follows that Corollaries 3.2 hold with $\omega_{1}$ replaced by $\lambda$ even when the 
relation " $\lambda$-PI" is restricted to Boolean algebras.]

It remains to show that $\mathbf{B}_{\mathbf{0}} \approx \mathbf{B}_{1}$. We could prove this directly but prefer to state a more general theorem of independent interest, which is analogous to Theorem 5.2. In this theorem $\kappa$ is not necessarily regular and for $A \subseteq \kappa^{+}$the ordering $\Phi(A)$ is defined as in $\$ 4$ (with the order type $\eta$ as in Proposition 4.1). The reader should verify that the nonisomorphism of $\mathbf{B}_{0}, \mathbf{B}_{1}$ mentioned above is a special case of the theorem.

6.5. Theorem. Let $A, B \subseteq \kappa^{+}$subject to the assumptions of Theorem 5.2. Then

$$
\mathrm{B}_{\Phi(A)} \simeq \mathrm{B}_{\Phi(B)} \text { iff } A \cap C=B \cap C
$$

for some closed unbounded $C \subseteq \kappa^{+}\left(\mathbf{B}_{\mathbf{O}}\right.$ is the interval algebra of $\left.\mathbf{O}\right)$.

Proof. If $A \cap C=B \cap C$ for some closed unbounded $C$ then, by 5.2, $\Phi(A) \cong \Phi(B)$ hence $\mathbf{B}_{\Phi(A)} \cong \mathbf{B}_{\Phi(B)}$. For the converse, let $\mathbf{B}_{1}=\mathbf{B}_{\Phi(A)}, \mathbf{B}_{2}=$ $\mathbf{B}_{\Phi(B)}$ and suppose that $\mathbf{B}_{1} \simeq \mathbf{B}_{2}$ by isomorphism $h$. Note that an element $b \in B_{1}$ is bounded (from above, as a subset of $\Phi(A)$ ), iff there is no collection of $\kappa^{+}$pairwise-disjoint elements of $\mathbf{B}_{1}$ which are $<b$; similarly for $\mathbf{B}_{2}$ and $\Phi(\mathbf{B})$. Thus the isomorphism $h$ preserves boundedness and so does its inverse $h^{-1}$.

Each of $\Phi(A), \Phi(B)$ consists of $\kappa^{+}$blocks (each of order type $\eta$ or $1+\eta$ ). Denote by $a_{0}\left(b_{0}\right)$ the first element of $\Phi(A)(\Phi(B))$ and by $\Phi_{\alpha}(A)\left(\Phi_{\alpha}(B)\right)$ the union of the first $\alpha$ blocks of $\Phi(A)\left(\Phi(B)\right.$ respectively) for $\alpha<\kappa^{+}$. We shall say that $h$ "takes $\Phi_{\alpha}(A)$ into $\Phi_{\beta}(B)$ " when for each $x \in \Phi_{\alpha}(A)$ there is some $y \in \Phi_{\beta}(B)$ such that $h\left[a_{0}, x\right) \subseteq\left[b_{0}, y\right)$. Similar terminology will be used for $h^{-1}$.

By using the above remark on preservation of boundedness and going back and forth $\omega$ times between $\Phi(A)$ and $\Phi(B)$ one sees that there is a closed unbounded set $C \subseteq \kappa^{+}$such that for each $\gamma \in C, h$ takes $\Phi_{\gamma}(A)$ into $\Phi_{\gamma}(B)$ and $h^{-1}$ takes $\Phi_{\gamma}(B)$ into $\Phi_{\gamma}(A)$. Now let $\gamma \in C$. If $\gamma \in A$ let $a_{\gamma}$ be the first element of the $\gamma$ th block of $\Phi(A)$. It is easily seen that $h\left[a_{0}, a_{\gamma}\right)=\Phi_{\gamma}(B)$ so that the $\gamma$ th block of $\Phi(B)$ must have a first point (otherwise $\Phi_{\gamma}(B) \notin B_{2}$ ), hence $\gamma \in B$. Similarly $\gamma \in B \Rightarrow \gamma \in A$. We have thus shown that $A \cap C=$ $B \cap C$ for some closed unbounded $C \subseteq \kappa^{+}$.

7. A stronger notion of potential isomorphism. In this section $\mathbf{M}$ is a fixed countable standard model of ZFC and $\kappa, \lambda, \ldots$ denote infinite cardinals of M. So far we have concentrated (except in Theorems 2.5 and 2.7) on one notion of potential isomorphism " $\lambda$-PI over $M$ " and proved that it is not well-behaved. A slight variant of this notion is the following: For $\mathbf{A}, \mathbf{B} \in M$ let " $A(\lambda-P I) B$ over $M$ " mean that there exists a Cohen extension $\mathbf{N}$ of $\mathbf{M}$ such that $N \cap^{<\lambda} M \subseteq M$ and $\mathrm{A}$ and $\mathrm{B}$ are isomorphic in $\mathbf{N}$. This concept can be defined within $\mathbf{M}$; in fact by standard techniques of forcing and Boolean valued models (cf. [Je1] whose notation and terminology we follow except that for us $p>q$ means that condition $p$ is stronger (more informative) than 
q) we get:

$\mathbf{A}\left(\lambda-\mathrm{PI}^{\prime}\right) \mathbf{B}$ over $\mathbf{M}$ iff $\mathbf{M}$ satisfies: "There exists a nondegenerate $(<\lambda$, $<\infty)$-distributive complete Boolean algebra $\mathbf{C}$-such that $V^{(\mathbf{C})} \vDash \check{\mathbf{A}} \cong \dot{\mathbf{B}}$ ". Also, $\mathbf{A}\left(\lambda-\mathbf{P I}^{\prime}\right) \mathbf{B}$ over $\mathbf{M}$ iff $\mathbf{M}$ satisfies: "There exists a $\lambda$-dense poset $\mathbf{P}$ such that some $p \in P$ forces that $A$ is isomorphic to B". (A poset (partially ordered set) $\mathbf{P}$ is said to be $\lambda$-dense when the intersection of any family of fewer than $\lambda$ dense open subsets of $P$ is dense.)

It turns out that the relation $\lambda$-PI' shares all the bad properties we have proved of $\lambda$-PI. This is clear since in all previous sections we could restrict ourselves to Cohen extensions of $\mathbf{M}$ without changing anything else.

We shall now consider a stronger relation of potential isomorphism which is much better behaved.

7.1. Definition. (a) A poset $\mathbf{P}$ is said to be $\lambda$-closed when any increasing sequence of elements of $\mathbf{P}$ whose length is $<\lambda$ has an upper bound in $\mathbf{P}$.

(b) " $A\left(\lambda-\mathrm{PI}^{*}\right) \mathbf{B}$ over $\mathbf{M}$ " when there exists a $\lambda$-closed poset $\mathbf{P}$ in $\mathbf{M}$ and a Cohen extension $\mathbf{N}$ of $\mathbf{M}$ via $P$ (that is $-\mathbf{N}=\mathbf{M}[G]$ where $G$ is some $\mathbf{M}$-generic filter over $\mathbf{P}$ ) such that $\mathbf{A}$ and $\mathbf{B}$ are isomorphic in $\mathbf{N}$.

We remark that every $\lambda$-closed poset is $\lambda$-dense (see, e.g., [Je1, p. 66]) and that the standard constructions of Cohen extensions $\mathbf{N}$ of $\mathbf{M}$ such that $N \cap^{<\lambda} M \subseteq M$ are by means of $\lambda$-closed posets (cf. [Je1, pp. 70-71, Model V]). In fact, the existence of $\lambda$-dense posets which are not $\lambda$-closed is not obvious (but it is true; it follows from the work of Gregory and Harrington [GH] that if $\lambda>\omega$ there exist Cohen extensions of $\mathbf{M}$ via $\lambda$-dense posets which cannot be obtained via $\lambda$ closed posets; a better-known example of such a poset is a $\lambda$-Souslin tree, if $\lambda$ is regular and such a tree exists).

The interest of the notion $\lambda-\mathrm{PI}^{*}$ is that it shares three basic properties of the standard potential isomorphism relation $\omega$-PI discussed in the introduction.

7.2. THEOREM. (a) The relation " $\lambda$-PI" over $\mathbf{M}^{*}$ is an equivalence relation.

(b) If in $\mathbf{M} \mathbf{A}$ and $\mathbf{B}$ are structures of cardinality $<\lambda$ and $\mathbf{A}\left(\lambda-\mathrm{PI}^{*}\right) \mathbf{B}$ over $\mathbf{M}$ then $\mathbf{A}$ and $\mathbf{B}$ are isomorphic in $\mathbf{M}$.

(c) If $\mathbf{A}\left(\lambda-P I^{*}\right) \mathbf{B}$ over $\mathbf{M}$ then $\mathbf{A}$ and $\mathbf{B}$ are isomorphic in every extension $\mathbf{N}$ of $\mathbf{M}$ via a $\lambda$-closed poset such that $\mathbf{N} \vDash|A|<\lambda,|B|<\lambda$.

Proof. (a) We need only prove transitivity. Assume that $\mathrm{A}_{1}\left(\lambda-\mathrm{PI}^{*}\right) \mathbf{A}_{2}$ and $\mathrm{A}_{2}\left(\lambda-\mathrm{PI}^{*}\right) \mathrm{A}_{3}$, over $M$. Find $\lambda$-closed posets $\mathbf{P}, \mathbf{Q}$ in $M$ and elements $p \in P$, $q \in Q$ such that $p \Vdash-\mathbf{A}_{1} \simeq \mathrm{A}_{2}$ and $q \Vdash \mathbf{A}_{2} \simeq \mathbf{A}_{3}$. Now let $K$ be an M-generic filter over the $\lambda$-closed poset $\mathbf{P} \times \mathbf{Q}$ containing $(p, q)$. By the Product Theorem [Sh, 88] we may write $M[K]=M[G][H]$ where $K=G \times H, G$ is M-generic over $\mathbf{P}$ and $H$ is $\mathbf{M}$-generic (even $M[G]$-generic) over $\mathbf{Q} ; p \in G$, $q \in H$. Thus in $\mathbf{M}[G], \mathbf{A}_{1} \approx \mathbf{A}_{2}$ and in $\mathbf{M}[H], \mathbf{A}_{2} \approx \mathbf{A}_{3}$ hence in $\mathbf{M}[K]$, $A_{1} \simeq A_{2} \simeq A_{3}$. This shows that $A_{1}\left(\lambda-P^{*}\right) A_{3}$.

(b) The proof is most conveniently stated in terms of Boolean valued models. By $R O(\mathrm{P})$ we mean the complete Boolean algebra (c.B.a.) naturally 
associated with $\mathbf{P}$ as in [Je1, p. 50]. We shall call a c.B.a. $\mathbf{C} \lambda$-special when $\mathbf{C}=R O(\mathbf{P})$ for some $\lambda$-closed nonempty poset $\mathbf{P}$. The following lemma is in fact known.

Lemma. Let $\mathbf{C}$ be a $\lambda$-special c.B.a. Then for any collection of $<\lambda$ joins and meets in $\mathbf{C}$ there is an ultrafilter preserving all of them.

Proof. Let $\mathrm{C}=R O(\mathrm{P})$ where $\mathrm{P}$ is $\lambda$-closed. We may assume that the given joins and meets are all joins and are, in fact, of the form

$$
\sum_{i \in I_{\alpha}} c_{\alpha i}=1 \quad(\alpha<\lambda)
$$

For each $\alpha<\lambda$ let

$$
D_{\alpha}=\left\{p \in P \mid\left(\exists i \in I_{\alpha}\right) \bar{p}<c_{\alpha i}\right\},
$$

where $\bar{p}$ is the element of $\mathbf{C}$ corresponding to $p$ in the natural mapping of $\mathbf{P}$ into C. Each $D_{\alpha}$ is a dense open set in $P$ (check this, using the fact that $\left.\Sigma_{i \in I_{a}} c_{\alpha i}=1\right)$ and since $P$ is $\lambda$-closed it is easy to construct a decreasing sequence $\left\langle p_{\alpha}\right| \alpha\langle\lambda\rangle$ such that $p_{\alpha} \in D_{\alpha}$ for each $\alpha$. Now $\left\langle\bar{p}_{\alpha}\right| \alpha\langle\lambda\rangle$ is a decreasing sequence of nonzero elements of $\mathbf{C}$ and hence there is an ultrafilter $U$ on $\mathbf{C}$ containing each $\bar{p}_{\alpha}$. Thus for $\alpha<\lambda$ we have $\left(\exists p \in D_{\alpha}\right) \bar{p} \in U$ hence

$$
\left(\exists i \in I_{\alpha}\right) c_{\alpha i} \in U,
$$

so that $U$ preserves the join $\Sigma_{i} c_{\alpha i}=1$.

We now prove part (b) of 7.2, assuming that the language of A, B has only relation symbols (this is clearly permissible).

Proof. Let $|A|,|B|<\lambda$ and assume $\mathbf{A}\left(\lambda-\mathrm{PI}^{*}\right) \mathbf{B}$ (over M). Thus, working in $\mathbf{M}$, we have $V^{(\mathbf{C})}=\ddot{\mathbf{A}} \simeq \check{\mathbf{B}}$ for some $\lambda$-special c.B.a. C. Thus

$$
V^{(\mathrm{C})}=(\exists R \subseteq \check{A} \times \check{\mathrm{B}})[R \text { is an isomorphism between } \check{\mathrm{A}} \text { and } \check{\mathrm{B}}] \text {. }
$$

By the maximum principle [Je1, p. 57, Lemma 50] choose some $R \in V^{(\mathrm{C})}$ such that

(1) $V^{(\mathbf{C})} \vDash R$ is an isomorphism between $\check{A}$ and $\check{\mathbf{B}}$.

For each $a \in A, b \in B$ let $r_{a b}=\|R(\check{a}, \check{b})\|$ (an element of $C$ ). From (1) we deduce the following:

(2) $\Sigma_{b} r_{a b}=1$ for each $a \in A$;

(3) $\Sigma_{a} r_{a b}=1$ for each $b \in B$;

(4) $r_{a b_{1}} \cdot r_{a b_{2}}=0$ if $b_{1} \neq b_{2}, r_{a_{1} b} \cdot r_{a_{2} b}=0$ if $a_{1} \neq a_{2}$.

For each relation symbol $P$ of the language we also have (if $P$ is $n$-ary):

(5) $r_{a_{1} b_{1}} \cdots r_{a_{n} b_{n}}=0$ if $\left(a_{1}, \ldots, a_{n}\right) \in P^{\wedge}$ and $\left(b_{1}, \ldots, b_{n}\right) \notin P^{B}$ or $\left(a_{1}, \ldots, a_{n}\right) \notin P^{\Lambda^{A}}$ and $\left(b_{1}, \ldots, b_{n}\right) \in P^{\mathbf{B}}$. Using the Lemma, let $U$ be an ultrafilter on $\mathbf{C}$ that preserves all the $(<\lambda)$ joins in (2), (3), and define $R^{\prime} \subseteq A \times B$ by: $R^{\prime}(a, b) \Leftrightarrow r_{a b} \in U$. Then (2), (3), (4), (5) and the fact that $U$ preserves arbitrary finite joins and meets show that $R^{\prime}$ is an isomorphism of $A$ and $B$. Note that the set of relation symbols may have any cardinality so 
the statement holds without restriction on the language of $\mathbf{A}, \mathbf{B}$. The proof of (b) is now complete.

(c) Suppose $\mathbf{A}\left(\lambda-\mathrm{PI}^{*}\right) \mathbf{B}$ over $\mathbf{M}$ and let $\mathbf{Q}$ be a $\lambda$-closed poset in $\mathbf{M}, q$ an element of $Q$ such that $q \vDash A \cong B$. Now consider any extension $\mathbf{N}_{1}=\mathbf{M}[G]$ where $G$ is $\mathbf{M}$-generic over a $\lambda$-closed poset $\mathbf{P}$ in $\mathbf{M}$ and $N_{1} \vDash|A|<\lambda$, $|B|<\lambda$. We have to show that $A$ and $B$ are isomorphic in $\mathbf{N}_{1}$.

Find an $\mathbf{N}_{1}$-generic filter $H$ over $\mathbf{Q}$ such that $q \in H$ and consider the extension $\mathbf{N}=\mathbf{N}_{1}[\mathbf{H}]=\mathbf{M}[G][H]$. Clearly $\mathbf{N} \supseteq \mathbf{M}[H]$. Since $q \in H, A$ and $B$ are isomorphic in $\mathbf{M}[H]$, hence in $\mathbf{N}$. But $\mathbf{N}$ is a Cohen estension of $\mathbf{N}_{1}$ via $\mathbf{Q}$, $\mathbf{Q}$ is a $\lambda$-closed poset in $\mathbf{N}_{1}$ (as it is $\lambda$-closed in $\mathbf{M}$ and $N_{1} \cap^{<\lambda} M \subseteq M$ ) and $A, B$ are structures of cardinality $<\lambda$ in $N_{1}$. Thus, applying part (b) of our theorem to $\mathbf{N}_{1}$ (rather than $\mathbf{M}$ ) $\mathbf{A}$ and $\mathbf{B}$ are isomorphic in $\mathbf{N}_{1}$.

We add some miscellaneous remarks on the relation $\lambda-\mathrm{PI}^{*}$. It is at least as strong as $\lambda$-PI hence (by Theorem 2.3) strictly stronger than $\equiv_{\infty \lambda}$ (for each $\lambda>\omega)$. For singular $\lambda$, every $\lambda$-closed poset is $\lambda^{+}$-closed so $\lambda$-PI* is equivalent to $\lambda^{+}$-PI* (also $\lambda$-PI $\Leftrightarrow \lambda^{+}$-PI as we saw in the proof of 2.3 ). If $\lambda=\kappa^{+}$, $\kappa=\kappa^{\kappa}$ then we know $\lambda$-PI is not transitive (even restricted to structures of cardinality $\lambda$ ) hence $\lambda-\mathrm{PI}^{*}$ is strictly stronger than $\lambda$-PI (even on structures of cardinality $\lambda$ ). If $V=L$ and $\lambda=\kappa^{+}$, again we know (5.7) that $\lambda-\mathrm{PI}^{*}$ is strictly stronger than $\lambda$-PI.

Problem. Is $\lambda-\mathrm{PI}^{*}$ strictly stronger than $\lambda$-PI for all $\lambda>\omega$ ? That is, given $\lambda>\omega$ in $\mathbf{M}$ do there always exist $\mathbf{A}, \mathbf{B} \in M$ such that $\mathbf{A}(\lambda-\mathrm{PI}) \mathbf{B}$ but not $\mathrm{A}\left(\lambda-\mathrm{PI}^{*}\right) \mathrm{B}$ (over $M$ )?

For other questions on $\lambda-\mathrm{PI}^{*}$ see the next section.

8. Remarks and problems. First a technical remark. By working slightly harder we could replace the usual assumption that $\mathbf{M}$ is a (countable) standard model of ZFC by the assumption (in each place as the need arises) that $M$ satisfies a suitable finitely axiomatized fragment of ZFC (and similarly consider extensions $\mathbf{N}$ which are models of a fragment). In this way our theorems are seen in ZFC (by the reflection principle) to apply nonvacuously even if there is no standard model. Other ways of achieving this aim will occur to anyone familiar with forcing.

Several open problems were stated in the preceding sections. Perhaps the most basic among them is to extend to inaccessible $\lambda$ the detailed information on the relation " $\lambda$-PI" which we have obtained for successor $\lambda$. Related to this is the problem of constructing linear orderings $\mathbf{A}, \mathbf{B}$ such that $N_{\lambda}(\mathbf{A}, \mathbf{B})$ for inaccessible $\lambda$.

There is a general class of problems which has not been mentioned before but is a natural outgrowth of our initial motivation and negative results. The most comprehensive problem in this class is to find abstract logics $E$ and notions PI of potential isomorphism (in the sense of Definition 2.4 or a similar sense) such that PI characterizes $\equiv \mathfrak{e}$. The standard example is that $\mathcal{E}=L_{\infty \omega}$ and PI is our $\omega$-PI. [To make the problem precise one may have to refer to a countable standard model $\mathbf{M}$ and require $\mathcal{L}$ to be given by a 
definition of its syntax and semantics in set theory (as in the absolute logics of [Ba1]) or look at the real universe $V$ and consider Boolean valued models $V^{(\mathrm{C})}$ as the extensions.]

The problem as formulated above probably admits many trivial solutions (one would guess that a "reasonable" equivalence relation on structures could be $\equiv_{\mathfrak{L}}$ for some artificially constructed $\mathfrak{E}$ ) but if we require $E^{2}$ to properly extend $L_{\infty}$ and to be "natural" or "interesting" then we do not know any examples. A more special problem would be to find a natural $\mathbb{E}$ for which $\equiv_{\mathfrak{E}}$ is the relation " $\omega_{1}-\mathrm{PI}$ " of $\$ 7$, or to consider any of the numerous abstract logics $\mathcal{L}$ that have been studied (cf. [Ba1], [Ba3], [MSS]) and ask if $\equiv_{\mathbb{E}}$ can be expressed as potential isomorphism in some sense.

Notes (Added February, 1976). Since the completion of the paper, two of the problems presented ir. $\$ 9$ have been solved. First $S$. Shelah proved (in ZFC) that for each regular $\lambda>\omega$,nd also for singular $\lambda$ where $\lambda^{n_{0}}=\lambda$ there exist linear orderings A, B such th.' $N_{\lambda}(\mathrm{A}, \mathrm{B})$. Using them he got the more general result that if $T$ is any nonsuperstable countable first-order theory and $\lambda$ is as above, then $T$ has two models related by $N_{\lambda}$. This includes, e.g., the theory of Abelian groups.

A second problem mentioned above is that of finding a logic corresponding to the equivalence relation $\omega_{1}-\mathrm{PI}^{*}$. The outline of a solution was again suggested by $S$. Shelah. We define an increasing family of logics $\left\langle E^{*} \lambda\right| \lambda$ is regular $\rangle$ such that $\mathbf{A} \equiv_{e_{i}^{*}} \mathbf{B}$ iff $\mathbf{A}\left(\lambda-\mathrm{PI}^{*}\right) \mathbf{B}$ for all $\mathbf{A}, \mathbf{B}$ and regular $\lambda$ (for singular $\lambda, \lambda-\mathrm{PI}^{*}$ is the same as $\left.\lambda^{+}-\mathrm{PI}^{*}\right)$. The language $L_{\lambda}^{*}$ is closely related to $L(\lambda)$ of Keisler [Ke] but instead of linearly-ordered (at most $\lambda$-like) quantifier strings we have "tree quantifiers" of height $<\lambda$ of the kind considered by Hintikka and Rantala [HR]. (A tree-quantifier is a tree whose nodes are labeled by $\wedge, \vee, \forall, \exists$ and the formulas are attached to its branches; satisfaction is defined using a game in which a branch is generated, with one player playing at $\Lambda$ and $\forall$, the other at $\vee$ and $\exists$.) The bridge between these languages and $\lambda-\mathrm{PI}^{*}$ is supplied by the following simple game-theoretic characterization: $\mathbf{A}\left(\lambda-\mathrm{PI}^{*}\right) \mathbf{B}$ iff player II has a winning strategy in the Ehrenfeucht game of length $\lambda$ between $\mathbf{A}$ and $\mathbf{B}$ (for regular $\lambda$ ). This observation leads also to a simple proof of Theorem 7.2(b).

These developments indicate, we believe, the naturalness of the relations $\lambda-\mathrm{PI}^{*}$ and perhaps also the fruitfulness of the idea of looking for new logics by studying equivalence relations between structures.

\section{REFERENCES}

[Ba1] K. J. Barwise, Absolute logics and $L_{\infty \omega}$, Ann. Math. Logic 4 (1972), 309-340. MR 49 \#2252.

[Ba2] __ Back and forth through infinitary logic, Studies in Model Theory (M. Morley, ed.), MAA Studies in Math., vol. 8, Math. Assoc. Amer., Buffalo, New York, 1973, pp. 5-34. MR 49 \#1116.

[Be3] ___ Axioms for abstract model theory, Ann. Math. Logic 7 (1974), 221-265.

[Be] M. Benda, Reduced products and nonstandard logics, J. Symbolic Logic 34 (1969), 424-436. MR 40 \#092. 
[BHK] J. Baumgartner, L. Harrington and E. M. Kleinberg, Adding a closed unbounded set, J. Symbolic Logic 41 (1970), 481-482.

[Ch] C. C. Chang, Some remarks on the model theory of infinitary languages, The Syntax and Semantics of Infinitary Languages (J. Barwise, ed.), Lecture Notes in Math., vol. 72, SpringerVerlag, Berlin and New York, 1968, pp. 36-63.

[CK] C. C. Chang and H. J. Keisler, Model theory, North-Holland, Amsterdam, 1973.

[Co] J. Conway, Ph.D. Thesis, Cambridge, England, 196-.

[Ek] P. Eklof, On the existence of K-free abelian groups, Proc. Amer. Math. Soc. 47 (1975), 65-72.

[GH] J. Gregory and L. Harrington (unpublished).

[GS] H. Gaifman and E. P. Specker, Isomorphism types of trees, Proc. Amer. Math. Soc. 15 (1964), 1-7. MR 29 \# 5746.

[HR] J. Hintikka and V. Rantala, A new approach to infinitary languages, Ann. Math. Logic 10 (1976), 95-115.

[Hu] J. Hutchinson, Model theory via set theory (to appear).

[Je1] T. J. Jech, Lectures in set theory, with particular emphasis on the method of forcing, Lecture Notes in Math., vol. 217, Springer-Verlag, Berlin and New York, 1971. MR 48 \# 105.

[Je2] __ The axiom of choice, North-Holland, Amsterdam, 1973.

[Jn] R. B. Jensen, The fine structure of the constructible hierarchy, Ann. Math. Logic 4 (1972), 229-308. MR 46 \#8834.

[Ke] H. J. Keisler, Formulas with linearly ordered quantifiers, The Syntax and Semantics of Infinitary Languages (J. Barwise, ed.), Lecture Notes in Math., vol. 72, Springer-Verlag, Berlin and New York, 1968, pp. 96-130.

[MI] A. Mekler, Ph.D. Thesis, Stanford Univ., Calif., 1976.

[MSS] J. A. Makowski, S. Shelah and J. Stavi, $\Delta$-logics and generalized quantifiers, Ann. Math. Logic 10 (1970), 155-192.

[Na1] M. Nadel, Model theory in admissible sets, Ph.D. Thesis, Univ. of Wisconsin, 1971.

[Na2] _ Scott sentences and admissible sets, Ann. Math. Logic 7 (1974), 264-294.

[NS] M. Nadel and J. Stavi, $L_{\infty} \lambda^{-}$equivalence, isomorphism and potential isomorphism of structures, Notices Amer. Math. Soc. 22 (1975), p. A-644. Abstract \# 75T-E59.

[Pa] J. B. Paris, Solution to a problem of Max Dickman (to appear).

[Sa] G. E. Sacks, Saturated model theory, Benjamin, New York, 1972.

[Sh] J. R. Shoenfield, Unramified forcing, Axiomatic Set Theory (Proc. Sympos. Pure Math., vol. 13, Part I), Amer. Math. Soc., Providence, R.I., 1971, pp. 357-381. MR 43 \#6079.

[St1] J. Stavi, Superstationary sets and their applications (unpublished).

[St2] _ On $L_{\infty}$-equivalence of Boolean algebra rings and groups, Notices Amer. Math. Soc. 22 (1975), A-714. Abstract 75T-E77.

[Ta] W. W. Tait, Equivalence in $L_{\infty \lambda \lambda}$ and isomorphism (to appear).

\section{Department of Mathematics, Stanford University, Stanford, Calufornia 94305}

Current address (Mark Nadel): Department of Mathematics, Institute for Advanced Study, Princeton, New Jersey 08540

Current address (Jonathan Stavi): Department of Mathematics, Bar-Ilan University, RamatGan, Israel 